\title{
Intestinal microbiota shapes gut physiology and regulates enteric neurons and glia
}

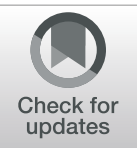

Fernando A. Vicentini 1,2,3,4, Catherine M. Keenan 1,2,4, Laurie E. Wallace ${ }^{1,2,4}$, Crystal Woods ${ }^{5}$, Jean-Baptiste Cavin 1,2,3, Amanda R. Flockton ${ }^{5}$, Wendy B. Macklin ${ }^{6}$, Jaime Belkind-Gerson ${ }^{5,7+}$, Simon A. Hirota ${ }^{2,3,4,8+}$ and Keith A. Sharkey ${ }^{1,2,4^{*}+}$ (D)

\begin{abstract}
Background: The intestinal microbiota plays an important role in regulating gastrointestinal (Gl) physiology in part through interactions with the enteric nervous system (ENS). Alterations in the gut microbiome frequently occur together with disturbances in enteric neural control in pathophysiological conditions. However, the mechanisms by which the microbiota regulates Gl function and the structure of the ENS are incompletely understood. Using a mouse model of antibiotic (Abx)-induced bacterial depletion, we sought to determine the molecular mechanisms of microbial regulation of intestinal function and the integrity of the ENS. Spontaneous reconstitution of the Abxdepleted microbiota was used to assess the plasticity of structure and function of the Gl tract and ENS. Microbiotadependent molecular mechanisms of ENS neuronal survival and neurogenesis were also assessed.
\end{abstract}

Results: Adult male and female Abx-treated mice exhibited alterations in Gl structure and function, including a longer small intestine, slower transit time, increased carbachol-stimulated ion secretion, and increased intestinal permeability. These alterations were accompanied by the loss of enteric neurons in the ileum and proximal colon in both submucosal and myenteric plexuses. A reduction in the number of enteric glia was only observed in the ileal myenteric plexus. Recovery of the microbiota restored intestinal function and stimulated enteric neurogenesis leading to increases in the number of enteric glia and neurons. Lipopolysaccharide (LPS) supplementation enhanced neuronal survival alongside bacterial depletion, but had no effect on neuronal recovery once the Abxinduced neuronal loss was established. In contrast, short-chain fatty acids (SCFA) were able to restore neuronal numbers after Abx-induced neuronal loss, demonstrating that SCFA stimulate enteric neurogenesis in vivo.

Conclusions: Our results demonstrate a role for the gut microbiota in regulating the structure and function of the Gl tract in a sex-independent manner. Moreover, the microbiota is essential for the maintenance of ENS integrity, by regulating enteric neuronal survival and promoting neurogenesis. Molecular determinants of the microbiota, LPS and SCFA, regulate enteric neuronal survival, while SCFA also stimulates neurogenesis. Our data reveal new insights into the role of the gut microbiota that could lead to therapeutic developments for the treatment of enteric neuropathies.

Keywords: Enteric nervous system, Gastrointestinal motility, Short-chain fatty acids, LPS, Enteric glia, Myenteric plexus, Submucosal plexus

\footnotetext{
* Correspondence: ksharkey@ucalgary.ca

${ }^{\dagger}$ Jaime Belkind-Gerson, Simon A. Hirota and Keith A. Sharkey contributed equally to this work.

'Hotchkiss Brain Institute, University of Calgary, Calgary, AB T2N 4N1, Canada

${ }^{2}$ Snyder Institute for Chronic Diseases, University of Calgary, Calgary, AB T2N

4N1, Canada

Full list of author information is available at the end of the article
}

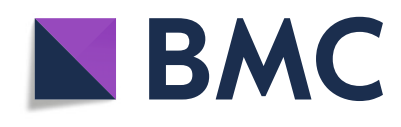

(c) The Author(s). 2021 Open Access This article is licensed under a Creative Commons Attribution 4.0 International License, which permits use, sharing, adaptation, distribution and reproduction in any medium or format, as long as you give appropriate credit to the original author(s) and the source, provide a link to the Creative Commons licence, and indicate if changes were made. The images or other third party material in this article are included in the article's Creative Commons licence, unless indicated otherwise in a credit line to the material. If material is not included in the article's Creative Commons licence and your intended use is not permitted by statutory regulation or exceeds the permitted use, you will need to obtain permission directly from the copyright holder. To view a copy of this licence, visit http://creativecommons.org/licenses/by/4.0/. The Creative Commons Public Domain Dedication waiver (http://creativecommons.org/publicdomain/zero/1.0/) applies to the data made available in this article, unless otherwise stated in a credit line to the data. 


\section{Background}

The gastrointestinal (GI) tract is regulated by finely tuned interactions of its cellular components, controlled by an intrinsic nervous system, the enteric nervous system (ENS) [1, 2]. The ENS is composed of two ganglionated plexuses, the myenteric and submucosal plexuses, containing neurons and enteric glial cells (EGC), connected by a dense network of nerve fibers and glial processes within the gut wall [1]. The ENS regulates GI motility, fluid secretion/absorption, immune function, mucosal growth and integrity, and intestinal permeability [1-5]. Structural and functional alterations in the ENS are linked to GI diseases, including irritable bowel syndrome and inflammatory bowel disease $[6,7]$. These debilitating conditions are increasing in incidence globally and have an enormous socioeconomic impact $[7,8]$.

The intestinal microbiota plays a fundamental role as a determinant of human health and is a critical factor that regulates GI physiology and pathophysiology [911]. Early-life alterations in the intestinal microbiota cause changes in gut physiology, which can lead to longlasting effects on overall health [12-15]. More recently, there has been a focus on understanding the role of the microbiota on GI function in the mature, adult animal, including the microbiota's influence on the structure and function of the ENS [16-27]. With the widespread use of probiotics [28], and the increasing application of fecal microbiota transplant therapy [29], it is evident that a better understanding of the impact of host-microbe interactions on GI physiology is required. The molecular mechanisms responsible for the interactions between the intestinal microbiota and the ENS are an area of active investigation. Two classes of molecules have emerged as playing important roles: ligands for toll-like receptors (TLR) and short-chain fatty acids (SCFA).

TLRs recognize microbial-associated molecular patterns (MAMPs) and are involved in host defense mechanisms of the innate immune system [30]. Recently, the role of TLRs has expanded beyond classical immune responses [31], with receptor expression being identified in other cell types, including in neurons of the ENS [18, $25,32,33]$. Recent reports suggest that TLRs regulate neuronal survival and neurogenesis $[18,25,32]$. MAMPs, such as lipopolysaccharide (LPS), are expressed by many classes of commensal enteric bacteria and are likely involved in the functional interactions of the microbiota and the ENS. In addition to MAMPs, bacterial products have also been shown to influence GI function and the structure of the ENS [34, 35]. SCFA are well-characterized bacterial products that have been demonstrated to affect GI physiology, through modulation of 5-hydroxytryptamine (5-HT; serotonin) [24, 36] and other mechanisms [37].
The role of these microbial determinants in regulating GI function and the structural integrity of the ENS in adult animals remains to be fully elucidated. Our goals were to determine the role of the microbiota and their molecular mediators (i.e., LPS and SCFA) on GI motility, intestinal permeability, fecal output, and the structural organization of the ENS, using a mouse model of antibiotic (Abx)-induced bacterial depletion. We sought to determine whether alterations in GI function and the ENS could be reversed upon reconstitution of the intestinal microbiota over the course of a 3-week Abx washout period. To determine the role of LPS and SCFA in the regulation of gut function and ENS structure, we supplemented Abx-treated mice with either LPS or SCFA at different timepoints to investigate the impact of these key host-microbe interactions.

\section{Results}

Abx-induced depletion of the intestinal microbiota alters the structure and function of the gut in a sexindependent manner

We first examined the effects of depleting the intestinal microbiota on GI morphology and physiology. We treated mice with an Abx regimen (Fig. 1a) that effectively reduces the enteric microbiota by over $99 \%$, as we have shown previously [38]. No alterations in body weight were observed over the course of Abx treatment (Fig. 1b). Interestingly, anatomical alterations were observed in both sexes in the cecum (Fig. 1c) and small intestine (Fig. 1d), but not in the colon (Fig. 1e), where the absolute number of bacteria are most abundant [39]. Abx-treated mice exhibited larger ceca (Fig. 1c) and an increase in small intestinal length (Fig. 1d) compared to non-Abx control mice. To determine whether the increased small intestinal length was due to tissue elongation, and not an effect of reduced contractile tone, tissues from both groups were treated with nifedipine, an L-type voltage-gated calcium channel blocker, to induce maximal muscle relaxation. Despite inducing relaxation with nifedipine, small intestinal tissues from Abxtreated mice were still significantly longer than control tissues (Figure S1), suggesting that the intestine lengthens to compensate for the loss of the microbiota.

To evaluate the effects of Abx treatment on intestinal function, we first assessed the fecal pellet output during a novel environment stress test. We also measured the water content in the expelled feces to detect any net alterations in secretion and/or absorption. In both male and female mice, Abx treatment induced higher fecal pellet wet weight compared to the control group (Fig. 1f), suggesting an enhanced response to stress in Abx-treated mice. Additionally, an increase in the fecal water content was observed in Abxtreated mice (Fig. 1g), suggesting changes in water absorption and/or secretion. Next, we evaluated GI motility using 


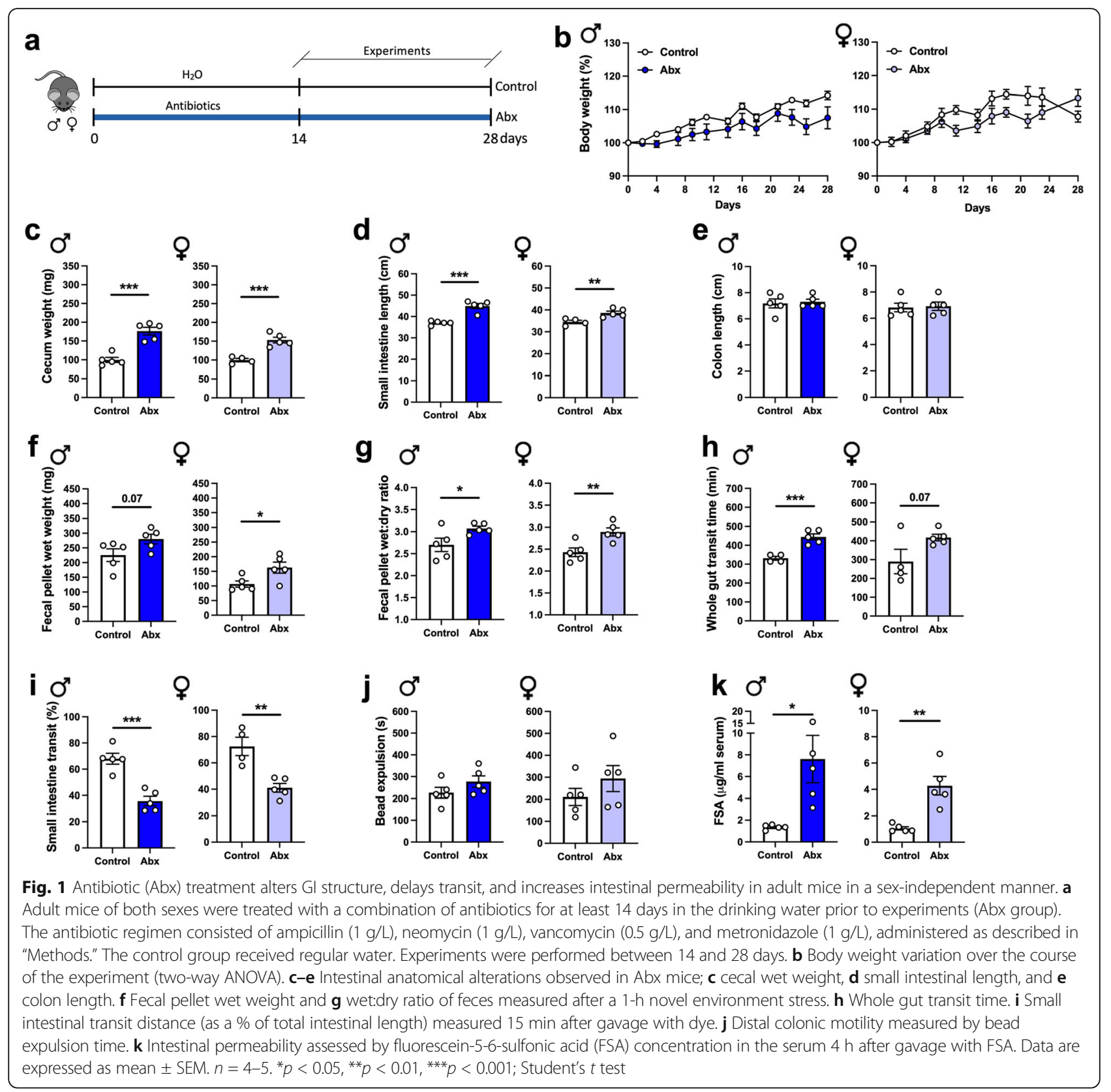

three different approaches. We found that GI motility was slowed in Abx-treated mice, indicated by an increase in whole gut transit time (Fig. 1h). To determine the origin(s) of the altered whole gut transit, we performed tests to evaluate small intestinal transit and distal colonic propulsion. Small intestinal motility, assessed by measuring dye migration along the intestine, was significantly reduced in Abx-treated mice (Fig. 1i). However, in the distal colon, we observed no differences in bead expulsion time between Abx-treated and control groups (Fig. 1j). No sex-dependent changes in motility were observed in Abx-treated mice (Fig. $1 h-j)$.
Next, we assessed intestinal barrier function by measuring fluorescein-5-6-sulfonic acid (FSA) leakage into serum [27]. Four hours following oral gavage, FSA was measured in the serum as an index of intestinal permeability. Abx-treated mice of both sexes exhibited increased serum FSA compared to control mice, suggesting that depletion of the intestinal microbiota increased intestinal permeability (Fig. 1k).

In summary, intestinal microbial depletion altered gut morphology, motility, and permeability in both male and female animals. 
Indiscriminate loss of enteric neurons is observed after depletion of gut bacteria, triggering enteric neurogenesis in the myenteric plexus

After observing alterations in GI morphology and function following Abx treatment (Fig. 1), we sought to determine if these events were associated with neuroanatomical changes in the ENS. Since we observed similar responses in male and female mice regarding Abx-induced alterations in GI function, we performed most of the remaining experiments in male mice. We first evaluated the neuroanatomical changes in two distinct intestinal regions, the distal ileum and proximal colon, analyzing both submucosal and myenteric plexuses (Fig. 2 and Figure S2). Strikingly, a loss of $\mathrm{HuC} / \mathrm{D}^{+}$ neurons in Abx-treated mice was observed in both the ileum (Fig. 2b, e) and the colon (Fig. 2c, f), in the submucosal and myenteric plexuses. This loss of enteric neurons was accompanied by a reduction in the Tuj $1^{+}$ neuronal fiber density (Figure S3a-b). By evaluating the two major subpopulations of enteric neurons, the $\mathrm{nNOS}^{+}$nitrergic and the ChAT ${ }^{+}$cholinergic neurons, we observed a loss of both the $\mathrm{nNOS}^{+}$neurons in the myenteric plexus (Fig. 2b, c; nNOS ${ }^{+}$neurons are virtually absent in the submucosal plexus) and $\mathrm{ChAT}^{+}$neurons in the submucosal and myenteric plexuses (Fig. 2e, f). To further explore the effect of Abx treatment in subpopulations of enteric neurons, we evaluated the impact of bacterial depletion on calretinin $\left(\mathrm{CALR}^{+}\right)$neurons (Figure S3c). After Abx treatment, $\mathrm{CALR}^{+}$neurons were reduced in the ileal submucosal and myenteric plexuses, and in the colonic submucosal plexus, but, interestingly, not in the colonic myenteric plexus (Figure S3d).

Additionally, we analyzed the effects of Abx treatment on enteric glia. We used two approaches to identify the EGC in our experiments (Fig. 3a, e), a PLP1-eGFPexpressing mouse [40], and the immunohistochemical expression of S100B; both well-established EGC markers $[41,42]$. We observed a complete overlap in the distribution of PLP1 and S100B expression (Figure S4), supporting their validity as markers of EGC. In PLP1-eGFP mice, we observed no alterations in the number of EGC cell bodies in the mucosa after the Abx treatment in either the ileum or colon (Fig. 3b). However, we detected a regional effect of intestinal microbial depletion on EGC in whole-mount preparations. A reduction in the PLP1-eGFP $^{+}$area (Fig. 3d), and a reduction in EGC number (Fig. 3f) were observed in the myenteric plexus in the ileum, but not the colon. The total number of EGC was not altered in the submucosal plexus of either region (Fig. 3f). Together, our data suggest a general loss of neurons in all regions analyzed after Abx-treatment, affecting neuronal subpopulations indiscriminately, and a reduction in the EGC population in the ileal myenteric plexus.
We next tested the hypothesis that the broad loss of neurons in the ENS after Abx treatment would trigger a compensatory response, potentially leading to increased enteric neurogenesis $[43,44]$. Sox 2 is a transcription factor known as a marker of ENS progenitor cells and is largely expressed by EGC in the adult animal $[45,46]$. Thus, we used $\mathrm{HuC} / \mathrm{D}^{+} / \mathrm{Sox}^{+}$double-labeling to evaluate the presence of Sox2-expressing neurons (Fig. 4), a putative marker of enteric neurogenesis $[45,46]$. We observed that the reduction of $\mathrm{HuC} / \mathrm{D}^{+}$neuronal numbers in the Abx-treated animals was accompanied by a reduction in $\mathrm{Sox}^{+}$cells in the colonic submucosal plexus (Fig. 4b). Surprisingly, we did not detect any $\mathrm{HuC} / \mathrm{D}^{+} /$ Sox $2^{+}$neurons in the submucosal plexus (Fig. 4b). In contrast, the decreased number of $\mathrm{HuC} / \mathrm{D}^{+}$neurons in the colonic myenteric plexus of Abx-treated animals was followed by an increase in $\mathrm{Sox} 2^{+}$cells and $\mathrm{HuC} / \mathrm{D}^{+} /$ Sox $2^{+}$neurons (Fig. 4d), indicative of increased neurogenesis in the colonic myenteric plexus of the microbiota-depleted mouse. Further, we tested whether cell proliferation was evident in the ENS by evaluating ileal and colonic sections stained for Ki67, a marker of cell proliferation. As expected, we observed $\mathrm{Ki} 67^{+}$cells at the base of the intestinal epithelial crypts, but we observed no positive staining in the ENS (Figure S5a). Moreover, we tested whether Abx treatment would lead to higher incorporation of 5-ethynyl-2'-deoxyuridine (EdU) into enteric cells, demonstrating de novo DNA synthesis. We did not detect positive EdU incorporation/ staining in the ENS (Figure S5b). Thus, Abx treatment triggered region-specific increased expression of Sox2 in neurons, which is indicative of enteric neurogenesis; however, no markers of proliferation were detected in enteric neurons.

\section{Spontaneous recolonization of bacteria restores altered GI physiology and ENS neuronal loss}

We next assessed if the alterations induced by intestinal microbial depletion could be reversed following cessation of Abx treatment. We allowed spontaneous microbial recolonization for 21 days (Fig. 5a). To confirm bacterial recolonization, we assessed the bacterial load in the feces of the different groups. Bacterial load in the Abx-withdrawal (microbiota recovery) group was comparable to the control group (Figure S6). The Abxwithdrawal led to a slightly lower body weight as compared to the other two groups by the end of the experiment (Fig. 5b). Alterations in gut morphology induced by Abx treatment were normalized following bacteria recolonization (Fig. 5c, d); Abx-withdrawal mice had reduced cecal weight (Fig. 5c) and shorter small intestinal length compared to the Abx-treated group (Fig. 5d). No changes in colon length were observed (Fig. 5e). The fecal pellet output in response to stress also returned to 


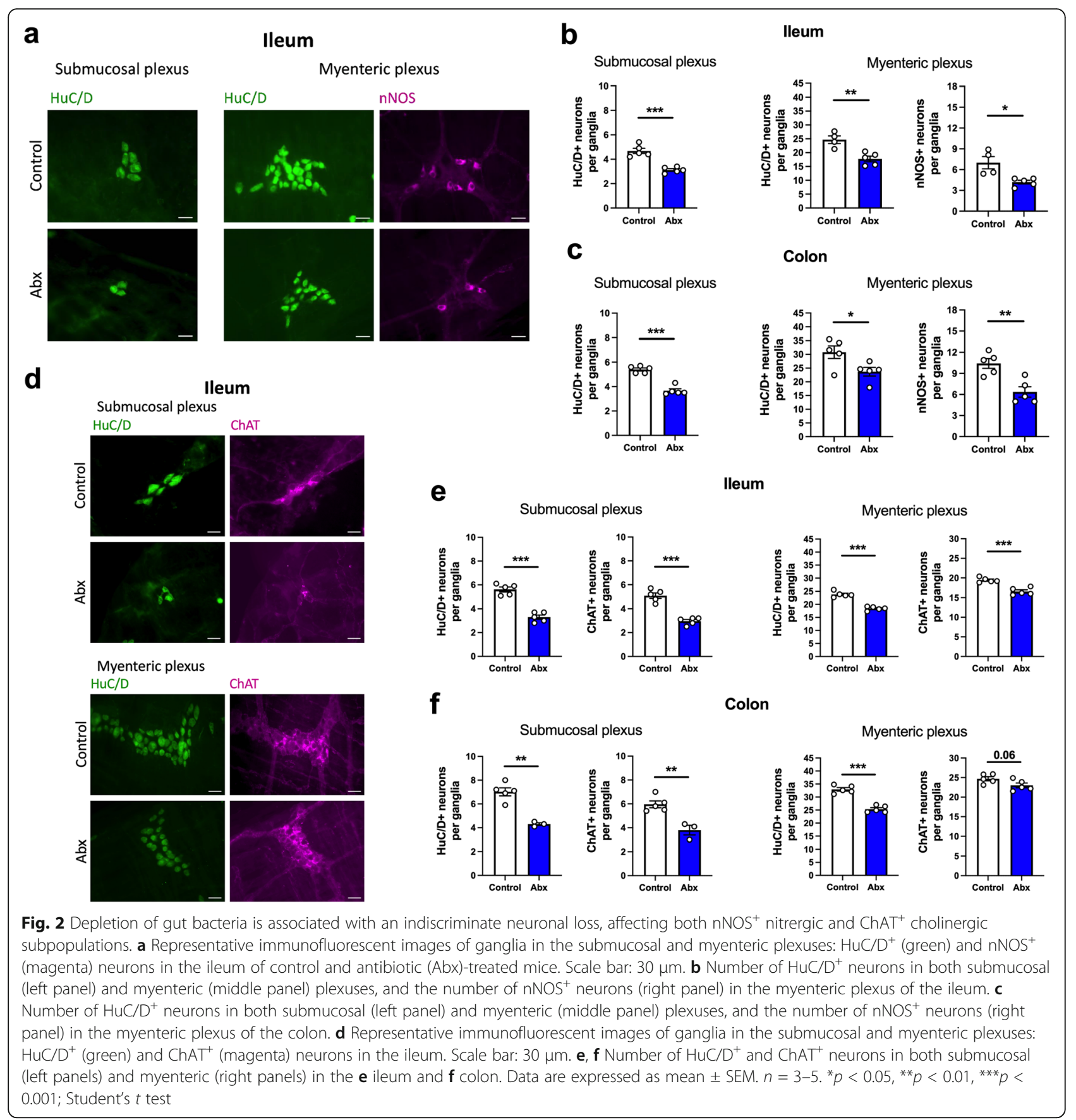

baseline levels after recolonization (Fig. $5 f$ ), with a tendency $(p=0.10)$ to recovery in the wet:dry ratio of the feces (Fig. 5g).

To further explore the effects of bacterial depletion and recovery on secretion, segments of distal ileum were collected and mounted in Ussing chambers. No differences in ion transport, as measured by changes in shortcircuit current $(\Delta \mathrm{Isc})$, were observed between $\mathrm{Abx}$ treated and Abx-withdrawal groups when tissues were treated with veratridine, a stimulator of nerve-mediated secretion (Fig. 5h, left panel). However, when tissues were treated with carbachol, to directly activate the intestinal epithelium, we observed increased $\Delta I s c$ in tissues isolated from Abx-treated mice, which was not observed in the Abx-withdrawal group (Fig. 5h, right panel). These results suggest a role for a bacteria-dependent regulation of intestinal ion transport by epithelial cells.

The motility patterns evaluated by three different tests also demonstrated a recovery to initial baseline levels after bacterial recolonization (Fig. 5i-k). Lastly, 


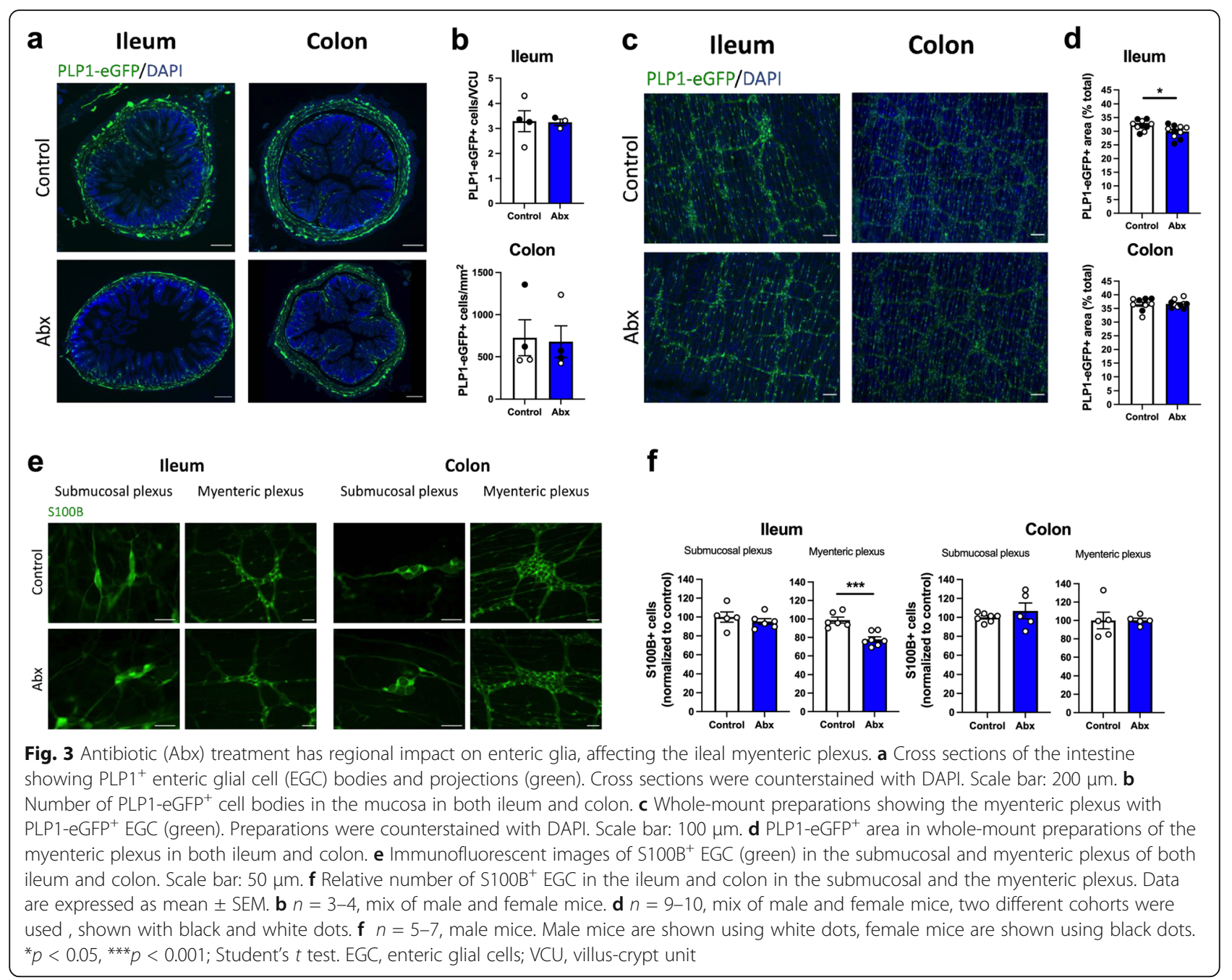

evaluating intestinal permeability, we observed an elevated concentration of FSA in the serum of Abx-treated mice, and this returned to baseline levels in the Abxwithdrawal group (Fig. 5l). Here, we also investigated whether the distal ileum may be contributing to the increased gut permeability of the Abx-treated mice. We measured the transepithelial electrical resistance (TER) of the distal ileum in the Ussing chambers. Interestingly, no differences were observed among the three groups (control: $27.17 \pm 1.46 \Omega / \mathrm{cm}^{2}$; Abx: $32.18 \pm 7.19 \Omega / \mathrm{cm}^{2}$; Abx-withdrawal: $31.39 \pm 3.01 \Omega / \mathrm{cm}^{2}$; one-way ANOVA). Overall, spontaneous reconstitution of the intestinal microbiota restored most of the altered physiological parameters induced by Abx treatment.

Next, we sought to determine the effect of the spontaneous bacterial recolonization on neurons and EGC of the ENS (Fig. 6). Given the normalization of many of the alterations in GI physiology driven by Abx treatment (Fig. 5), and the evidence for increased enteric neurogenesis after Abx treatment (Fig. 4), we hypothesized that intestinal microbial recolonization would restore the neuronal population in the ENS. Indeed, we observed a recovery of $\mathrm{HuC} / \mathrm{D}^{+}$neurons in the submucosal plexus and a recovery of $\mathrm{HuC} / \mathrm{D}^{+}$and $\mathrm{nNOS}^{+}$neurons in the myenteric plexus in the ileum of the Abx-withdrawal group (Fig. 6b). A similar tendency was observed in the colon regarding a restoration of $\mathrm{HuC} / \mathrm{D}^{+}$and $\mathrm{nNOS}^{+}$neurons in the Abx-withdrawal group (Fig. $6 \mathrm{c}$ and Figure S7). The neuronal recovery was also accompanied by a complete normalization of the $\mathrm{S} 100 \mathrm{~B}^{+} \mathrm{EGC}$ in the ileal myenteric plexus (Fig. 6d, e), illustrating the plasticity of the ENS. Moreover, we tested the modulation of neurogenesis after the microbiota recovery (Fig. 6f, g) and observed the restoration of $\mathrm{HuC} / \mathrm{D}^{+}$neurons as expected, but also a sustained increase in $\mathrm{Sox}^{+}$cells and $\mathrm{HuC} / \mathrm{D}^{+} / \mathrm{Sox} 2^{+}$neurons in the $\mathrm{Abx}$ withdrawal group (Fig. 6g).

Overall, our data show that the presence of microbial communities in the gut is a major modulator of GI 


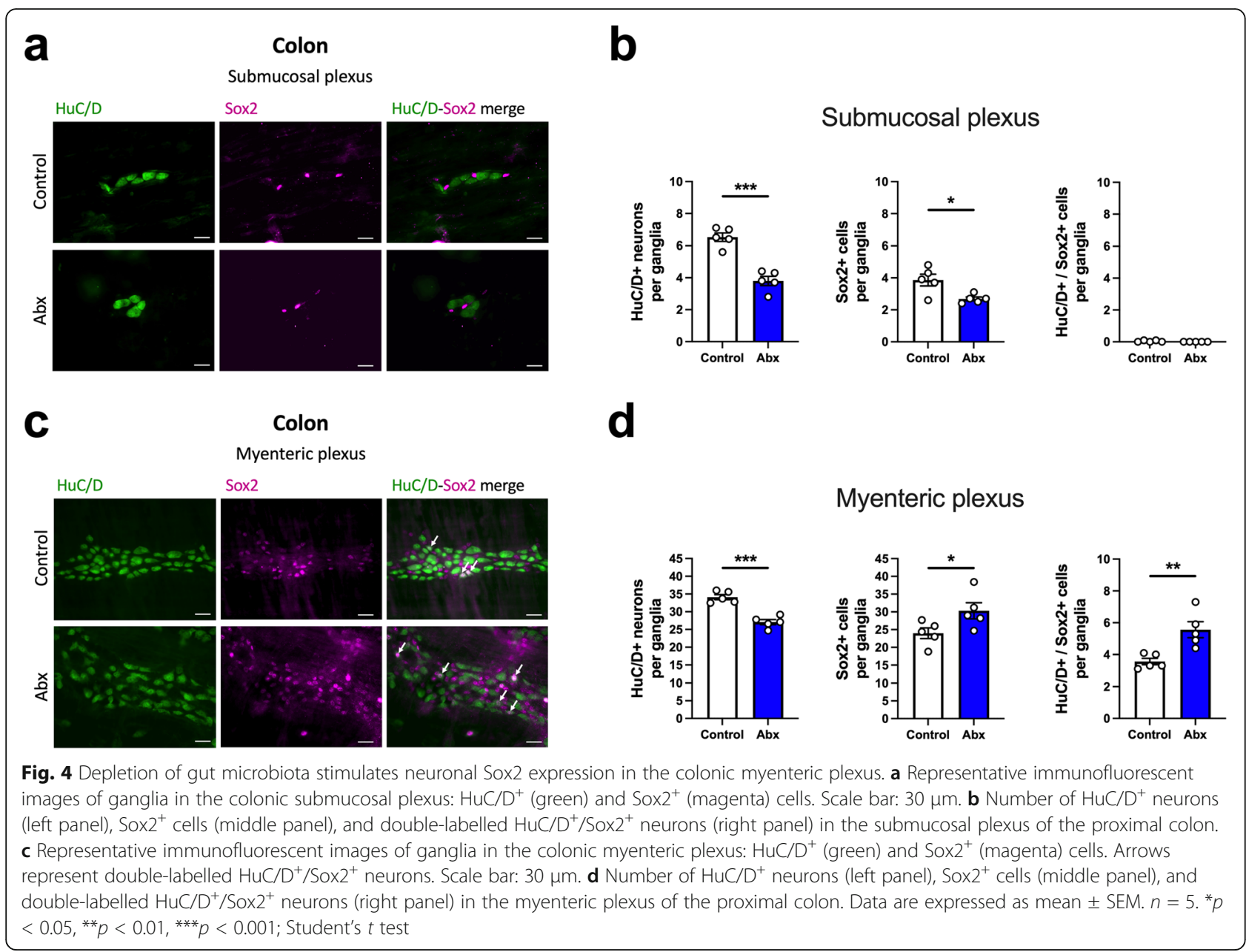

function and ENS integrity in the adult mouse, and that Abx-induced alterations in GI physiology and neuroanatomy of the ENS are largely reversible following spontaneous reconstitution of the microbiota.

\section{LPS supplementation prevents Abx-induced neuronal loss, but does not normalize GI function}

Next, we sought to investigate potential mechanisms by which the intestinal microbiota influences GI function and integrity of the ENS. LPS is a component of the cell wall of Gram-negative bacteria and is a known ligand of TLR4, a receptor previously described as important in the regulation of gut motility and ENS neuronal survival [18]. LPS levels are reduced after Abx treatment [18]. Hence, we sought to test whether supplementation with LPS $(50 \mu \mathrm{g} / \mathrm{mL})$ in the drinking water, at a concentration reported to modulate GI motility in mice [47], could attenuate or reverse the changes in GI function and ENS structure (Fig. 7a). Briefly, one group received LPS supplementation concomitant with the Abx treatment (LPS group), and a second received LPS supplementation after 14 days of Abx treatment (LPS at d14); a timepoint in which alterations caused by Abx treatment were fully established (Figs. 1, 2, and 3). No differences in body weight were detected among the different groups at the end of the experiment (Fig. 7b). Changes in gut morphology caused by Abx treatment, such as an enlarged cecum and longer small intestine, were not reversed by LPS supplementation (Fig. 7c-e). Functionally, LPS supplementation had no significant effects on fecal pellet wet weight after novel environment stress or the wet:dry ratio of the feces (Fig. $7 \mathrm{f}, \mathrm{g}$ ), nor did it normalize $\Delta \mathrm{Isc}$ induced by veratridine or carbachol (Fig. 7h). As observed in previous experiments, Abx treatment slowed motility (Fig. $7 \mathrm{i}-\mathrm{k})$, which was not affected by LPS supplementation. Lastly, LPS supplementation had no effect on the alterations in intestinal permeability or TER induced by depletion of the intestinal microbiota (Fig. 6i; TER at d14: control: $27.14 \pm 2.17 \Omega / \mathrm{cm}^{2}$; Abx: $29.36 \pm 2.77 \Omega / \mathrm{cm}^{2} ;$ Abx + LPS: $32.65 \pm 1.14 \Omega / \mathrm{cm}^{2}$; Abx + LPS: $35.85 \pm 1.31 \Omega / \mathrm{cm}^{2}$; one-way ANOVA). Taken together, our LPS supplementation regimens (concomitant with Abx administration or after 14 days of Abx treatment), did not normalize the Abxinduced changes in GI structure or physiology. 


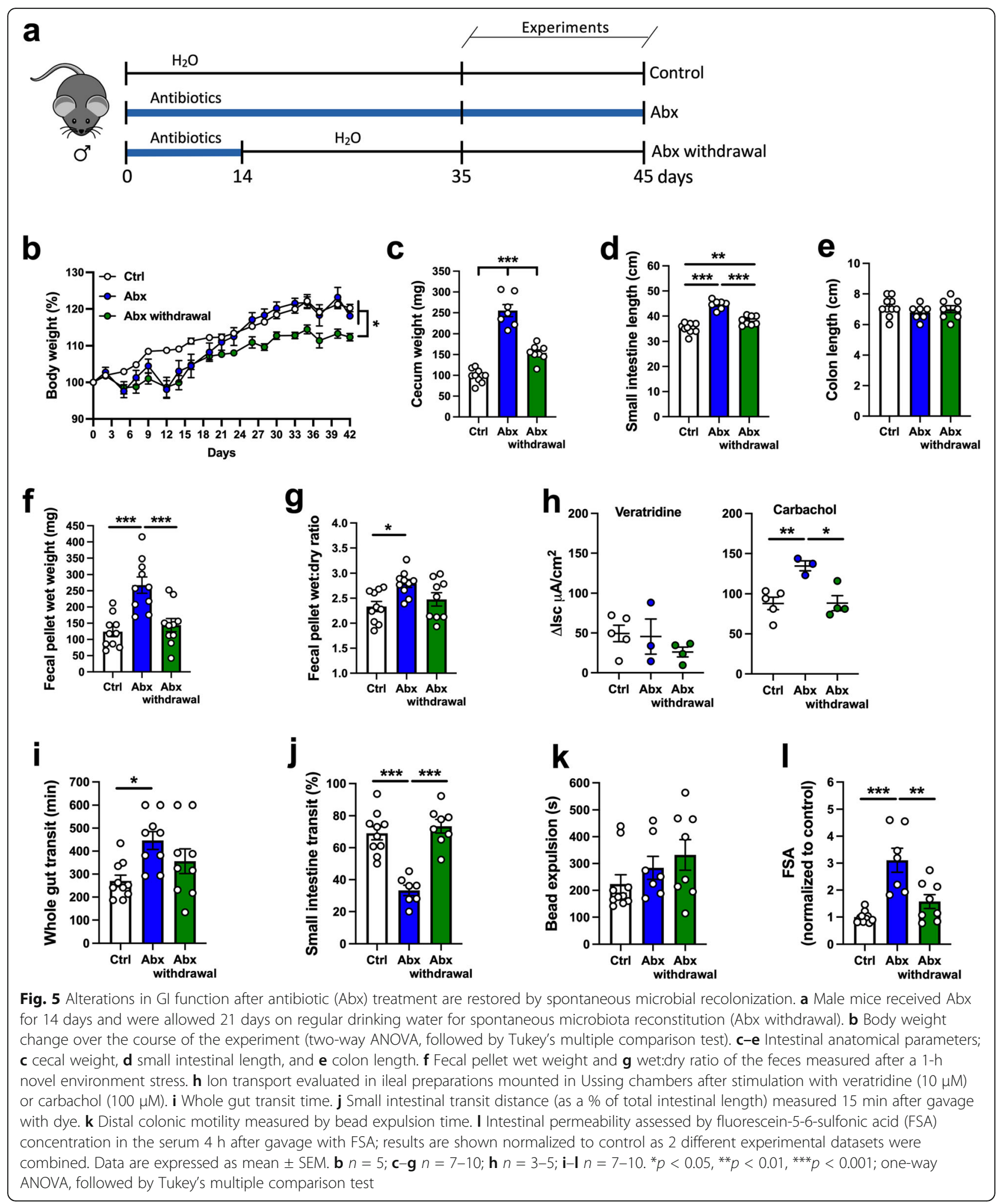

Although no significant changes in GI function were observed in Abx-treated mice supplemented with LPS, striking neuronal changes were observed (Fig. 8). In the ileum, Abx treatment reduced the number of $\mathrm{HuC} / \mathrm{D}^{+}$ neurons in both plexuses (Fig. 8a, left and middle panels), as observed in our previous experiments. Interestingly, concomitant administration of LPS with Abx prevented the loss of $\mathrm{HuC} / \mathrm{D}^{+}$neurons (Fig. 8a, left and 


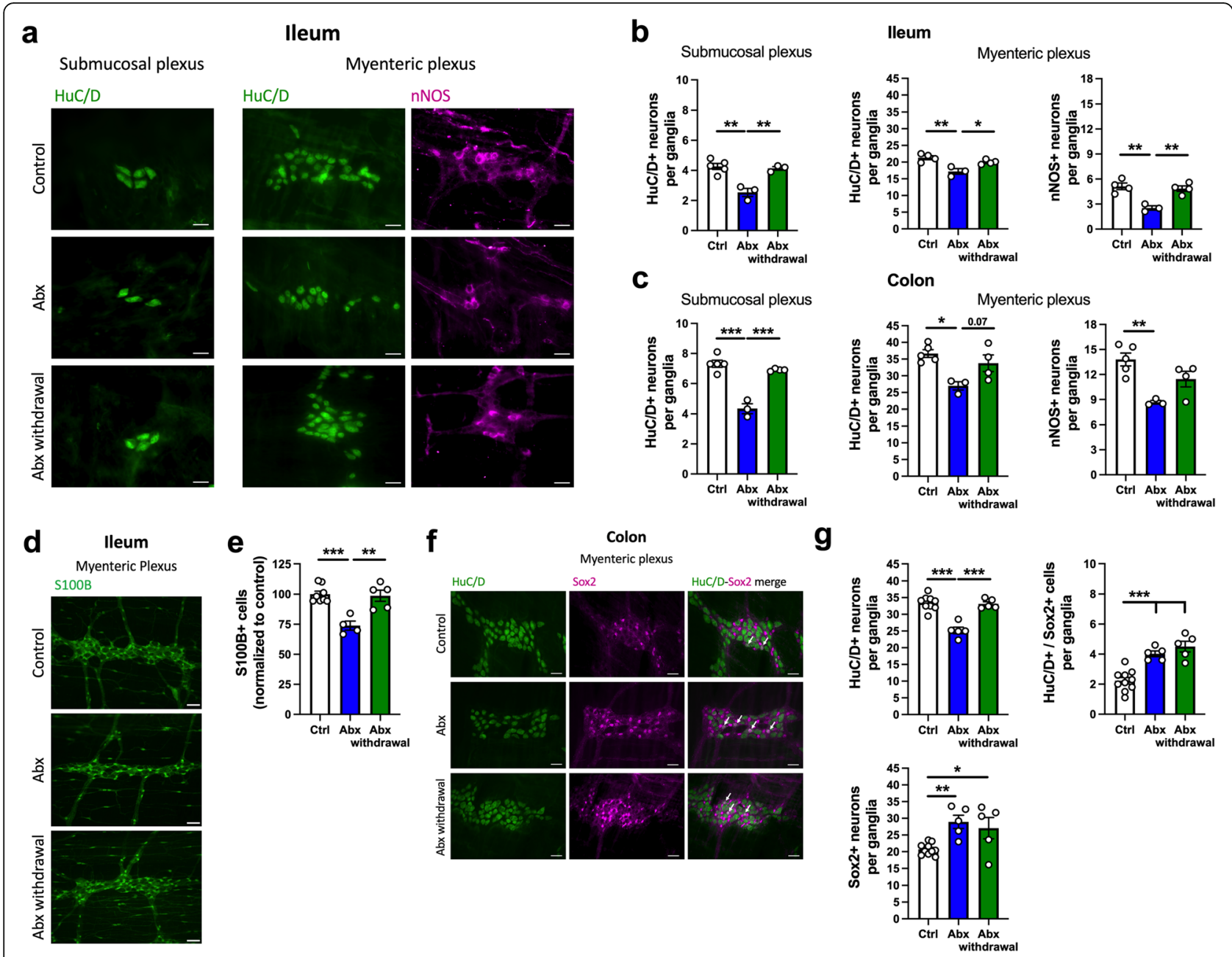

Fig. 6 Neuronal and glial loss induced by antibiotic (Abx) treatment are reversed by spontaneous microbial recolonization and are accompanied by enteric neurogenesis. a Representative immunofluorescent images of ganglia in the submucosal and myenteric plexuses: HuC/D $\left(\mathrm{green}^{+}\right.$and $\mathrm{nNOS}$ (magenta) neurons in the ileum. Scale bar: $30 \mu \mathrm{m}$. b Number of HuC/D+ neurons in the submucosal (left panel) and myenteric plexus (middle panel) in whole-mount preparations of the ileum. The number of nNOS neurons in the myenteric plexus (right panel) in the ileum. c Number of HuC/D+ neurons in the submucosal (left panel) and myenteric plexus (middle panel) in whole-mount preparations of the colon. The number of nNOS ${ }^{+}$ neurons in the myenteric plexus (right panel) in the colon. $\mathbf{d}$ Representative immunofluorescent images of ganglia in the ileal myenteric plexus showing S100B ${ }^{+}$enteric glial cells (EGC, green). Scale bar: $50 \mu \mathrm{m}$. e Relative number of S100B ${ }^{+}$EGC in the ileal myenteric plexus. f Immunofluorescence images of representative ganglia of the colonic myenteric plexus: HuC/D ${ }^{+}$(green) and Sox $2^{+}$(magenta) cells. Arrows represent

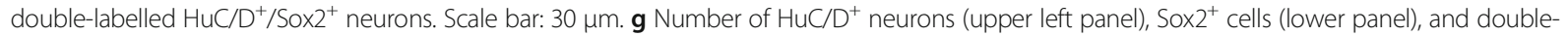
labelled HuC/D $/$ Sox $2^{+}$neurons (upper right panel) in the myenteric plexus of the proximal colon. Data are expressed as mean \pm SEM unless stated otherwise. $\mathbf{a}-\mathbf{c} n=3-5 ; \mathbf{d}-\mathbf{g} n=5-10 .{ }^{*} p<0.05,{ }^{* *} p<0.01,{ }^{* * *} p<0.001$; one-way ANOVA, followed by Tukey's multiple comparison test

middle panels). However, beginning LPS supplementation after 14 days of Abx treatment was not able to reverse the Abx-induced neuronal loss (Fig. 8a, left and middle panel). Moreover, a parallel effect was seen on the nitrergic neuronal subpopulation; $\mathrm{nNOS}^{+}$neurons were reduced in the Abx group compared to control, but this effect was attenuated with concomitant supplementation with LPS (Fig. 8a, right panel). Similar trends were observed in the colon (Fig. 8b). Both submucosal and myenteric plexuses had a reduction in the number of $\mathrm{HuC} / \mathrm{D}^{+}$neurons in the Abx-treated mice. This effect was attenuated in mice supplemented with LPS during
Abx treatment, but not when LPS was given after 14 days of Abx treatment (Fig. 8b, left and middle panels). However, the number of $\mathrm{nNOS}^{+}$neurons was not maintained by concomitant LPS supplementation in the colon (Fig. 8b, right panel). Together, these results suggest a role for LPS in neuronal survival, but not in ENS recovery (i.e., neurogenesis).

SCFA supplementation rescues neuronal loss induced by Abx treatment, but has a minor effect on Gl function Finally, we investigated the effects of SCFA supplementation in Abx-treated mice. The SCFA mix $(67.5 \mathrm{mM}$ of 


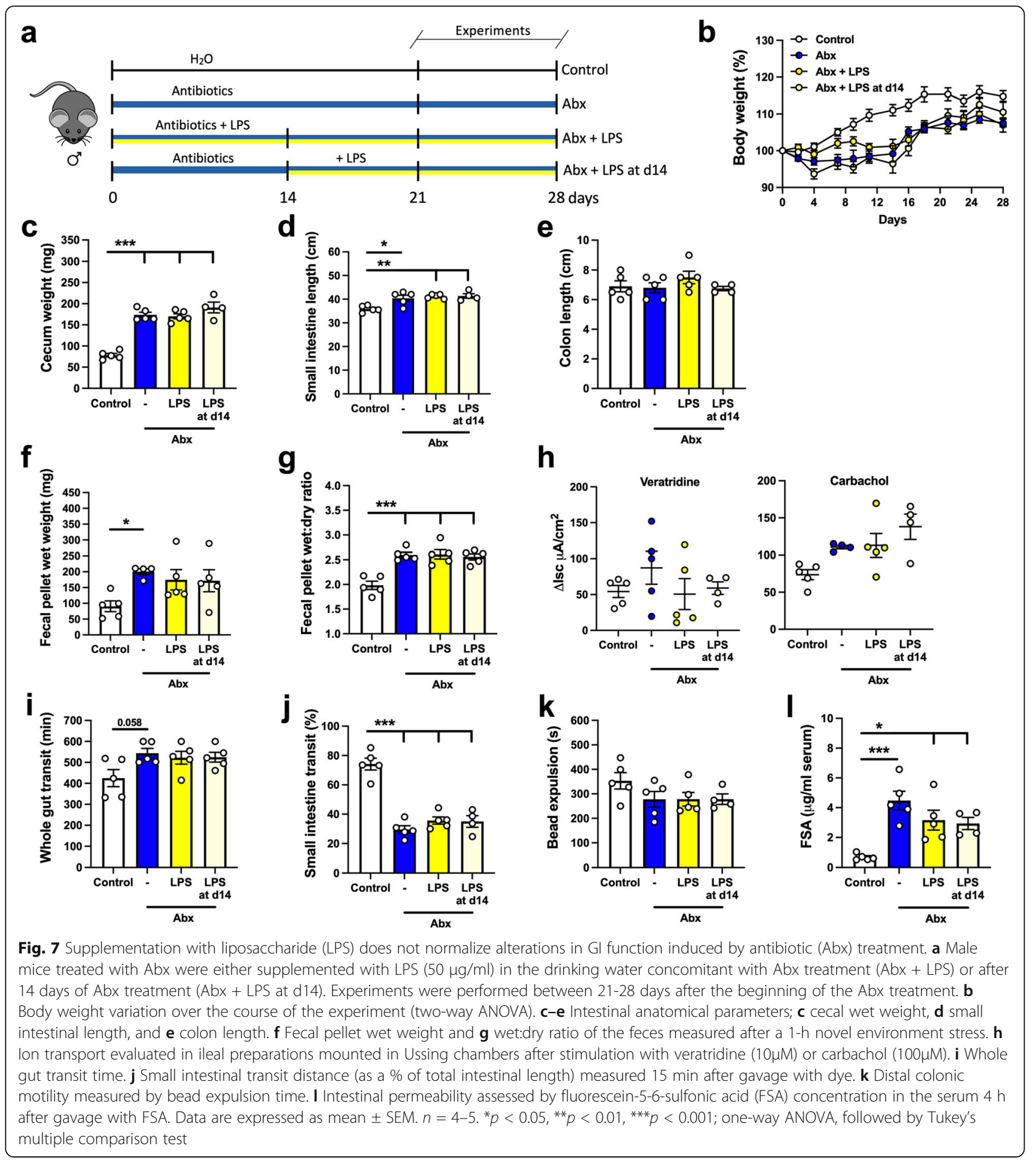

acetate, $25.9 \mathrm{mM}$ of propionate, $40 \mathrm{mM}$ of butyrate) [48] was administered in the drinking water concomitant with the Abx treatment (Abx + SCFA) or after 14 days of Abx treatment (Abx + SCFA at d14) (Fig. 9a).

A slightly reduced body weight was observed in the Abx group by the end of the experiment (Fig. 9b). Alterations in gut morphology caused by Abx treatment, such as enlarged cecum (Fig. 9c) and longer small intestine (Fig. 9d) were not affected by SCFA supplementation. No changes were observed in the colon length (Fig. 9e). SCFA supplementation, both concomitant and following 14 days of Abx treatment, reduced the elevated weight of fecal pellet output measured after the novel environment stress (Fig. 9f). The wet:dry ratio increase in the 


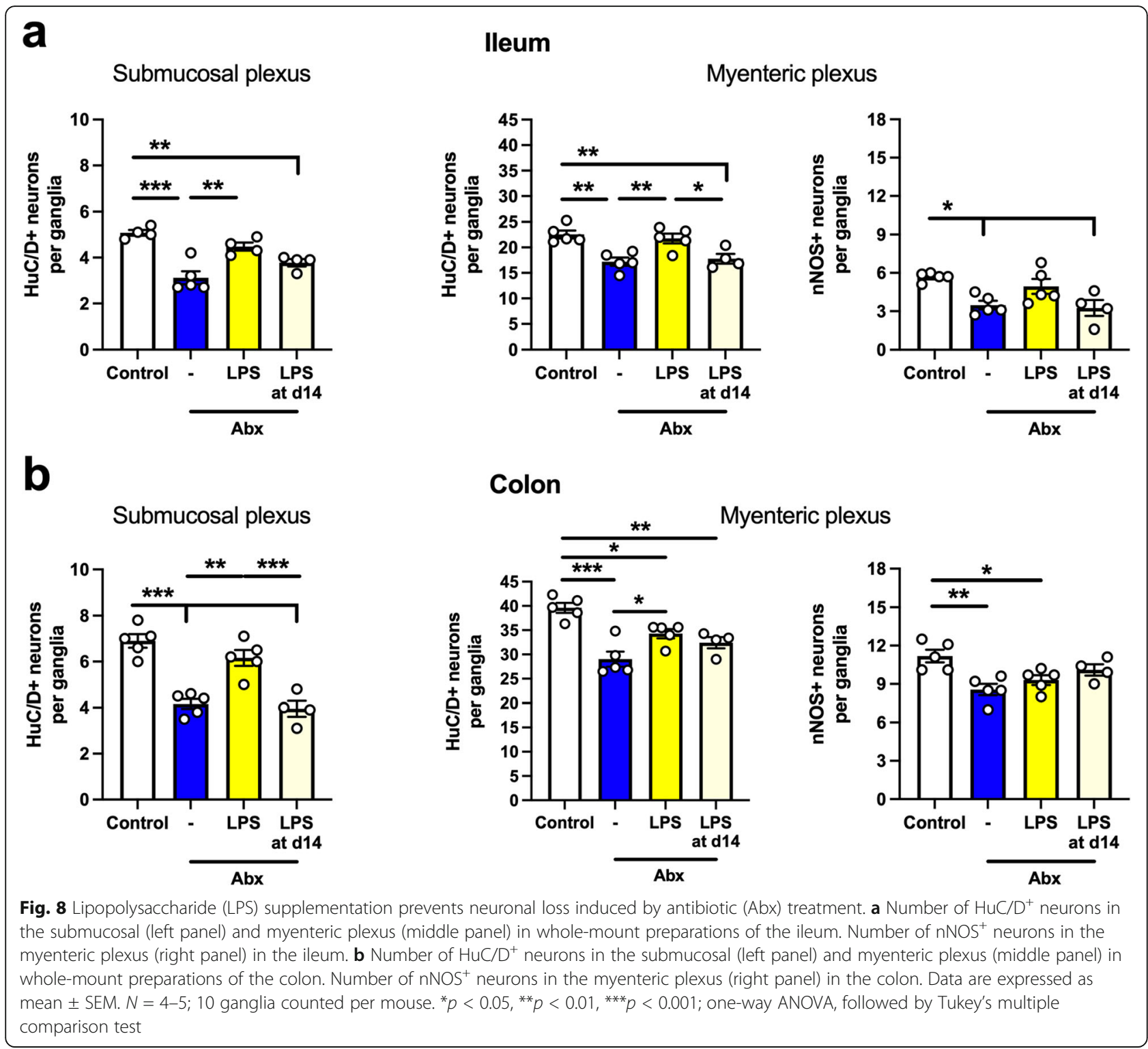

feces was not affected by SCFA treatment (Fig. 9g). No effects on ion transport in the ileum, nor motility or intestinal permeability, were observed in Abx-treated mice supplemented with SCFA (Fig. 9h-l; ileum TER at d14: control: $26.77 \pm 1.38 \Omega / \mathrm{cm}^{2}$; Abx: $35.84 \pm 3.91 \Omega / \mathrm{cm}^{2}$; Abx + SCFA: $33.25 \pm 2.98 \Omega / \mathrm{cm}^{2} ;$ Abx + SCFA: $37.75 \pm$ $2.49 \Omega / \mathrm{cm}^{2}$; one-way ANOVA).

Given the selective effects of SCFA supplementation on the functional parameters altered by Abx-induced intestinal microbiota depletion, we sought to determine if there was an effect on the neuroanatomical alterations in the ENS. Interestingly, a clear effect of the SCFA supplementation was observed in the submucosal plexus of both the ileum and the colon (Fig. 10a, b, left panels). A reduced number of $\mathrm{HuC} / \mathrm{D}^{+}$neurons was observed after Abx treatment, which was attenuated in both groups that received SCFA supplementation. In the ileal myenteric plexus, we observed neuronal recovery in mice supplemented with SCFA after 14 days of Abx treatment, but surprisingly, we did not observe a preservation of neurons when SCFA were delivered concomitant with Abx (Fig. 10a, middle panel). Additionally, no recovery effect was seen in the nitrergic $\mathrm{nNOS}^{+}$neuronal subpopulation (Fig. 10a, right panel). In the colonic myenteric plexus, mice treated with SCFA at both timepoints had a higher number of $\mathrm{HuC} / \mathrm{D}^{+}$and $\mathrm{nNOS}^{+}$neurons when compared to the Abx group (Fig. 10b, right panel). Due to this effect on neuronal recovery observed in the Abx + SCFA at the d14 group, we sought to evaluate whether SCFA would also regulate the number of $\mathrm{S} 100 \mathrm{~B}^{+} \mathrm{EGC}$ in the ileal myenteric plexus, which was the only region where EGC were sensitive to Abx treatment (Fig. 3f). 


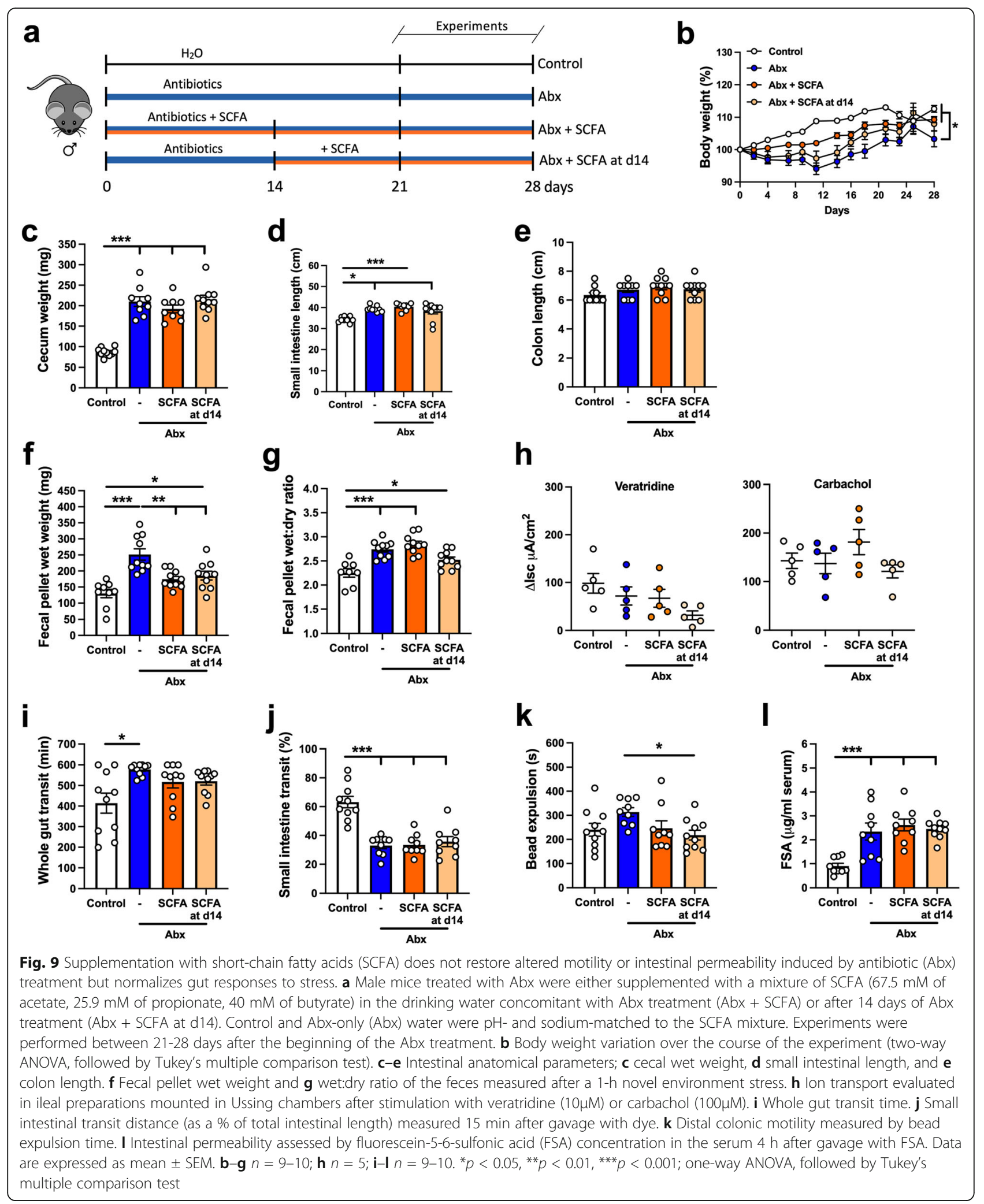

Here we observed a similar reduction in the $\mathrm{S} 100 \mathrm{~B}^{+}$ EGC numbers in the ileal myenteric plexus, however, no differences were observed after SCFA treatment $\left({\mathrm{S} 100 \mathrm{~B}^{+}}^{+}\right.$
EGC normalized to control: Control: $100 \pm 1.9$; Abx: $78.2 \pm 4.1$ ( $p<0.05$ compared to Control); Abx + SCFA: $87.2 \pm 6.3(p>0.05$ compared to Abx $) ; \mathrm{Abx}+$ SCFA at 


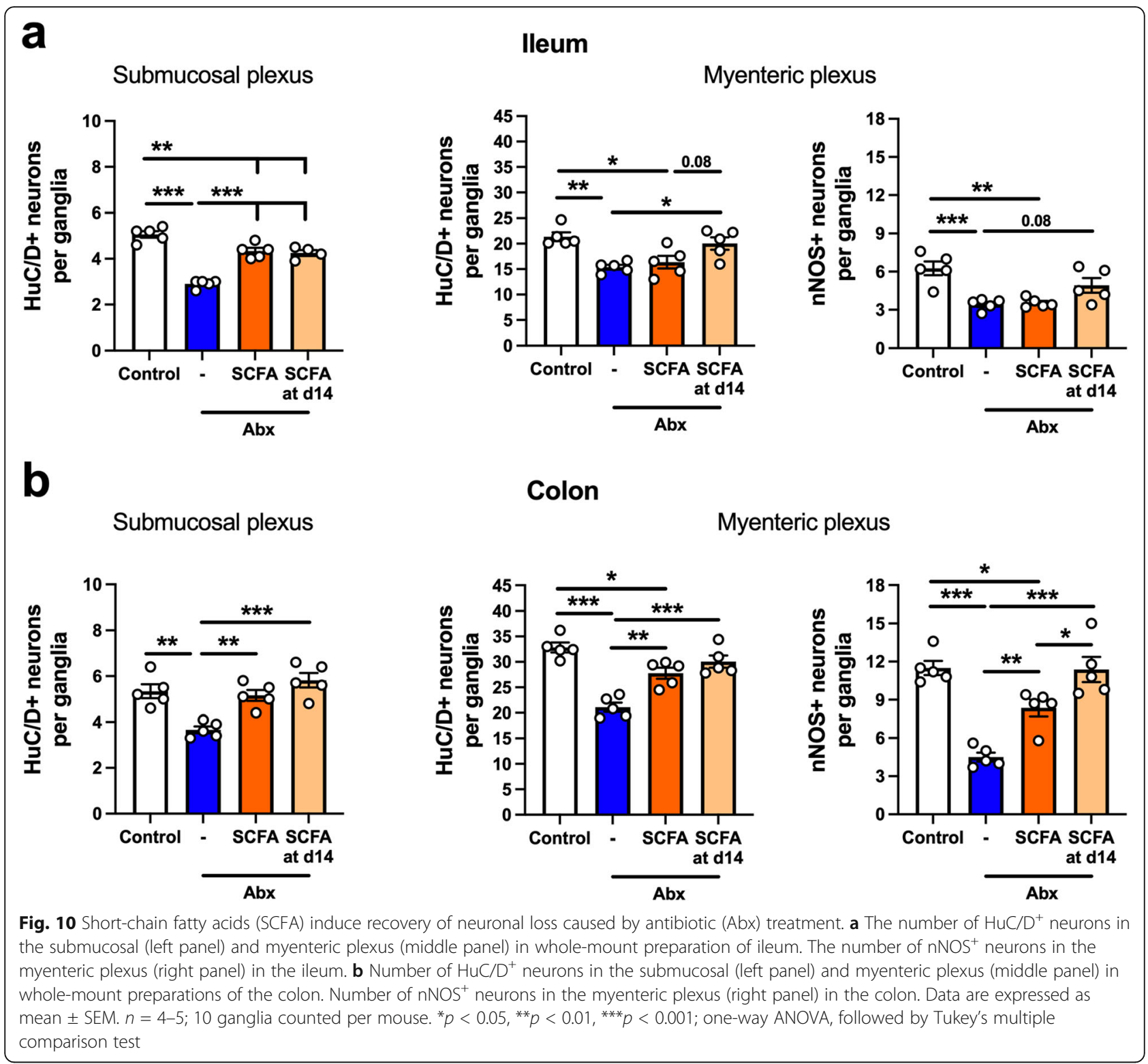

d14: $82.6 \pm 5.0$ ( $p>0.05$ compared to Abx); one-way ANOVA, followed by Tukey's multiple comparison test). These results suggest a specific effect of SCFA on enteric neurons.

Considering that SCFA restored the neuronal deficit induced by Abx treatment, but had only a minor effect on GI function, we asked whether a longer treatment regimen (90 days) with SCFA could restore the physiological alterations induced by $\mathrm{Abx}$ treatment (Fig. 11a). Morphological alterations induced by Abx treatment, such as increased cecum weight and longer small intestine length, were not restored by SCFA treatment (Fig. 11b, c). Moreover, long-term SCFA treatment did not restore the impaired motility induced by Abx treatment (Fig. 11d, e). We performed the whole gut transit test at two different timepoints, day 42 and day 77, and observed no difference between Abx and Abx+SCFA d14 groups (Fig. 11e). Similarly, no changes occurred after SCFA treatment when evaluating small intestine transit (Fig. 11d). Interestingly, SCFA restored the Abx-induced increase in intestinal permeability (Fig. 11e). These data suggest that SCFA may play a role in regulating barrier function, but their supplementation is insufficient to normalize most physiological functions perturbed by Abx treatment.

Together, we observed a role for SCFA in modulating enteric neurons in a region-specific manner, having the greatest effect in the submucosal plexuses and on colonic myenteric neurons. 


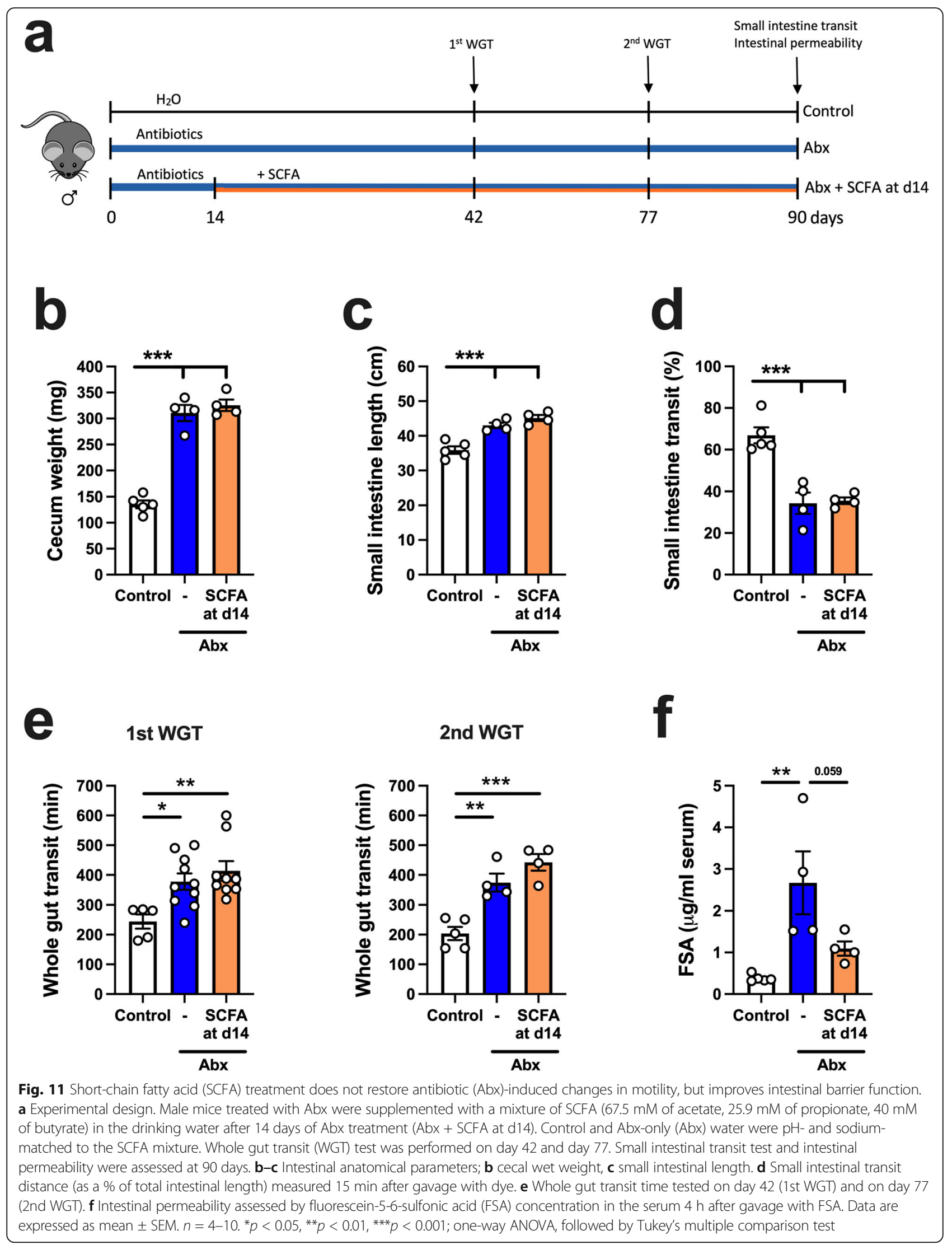




\section{Discussion}

In this study, we show the extent to which the intestinal microbiota regulates the structure and function of the GI tract in the adult mouse. We observed significant effects on small intestinal length, cecal mass, GI motility, ion transport, and intestinal permeability that were independent of the sex of the mice. Abx-treated mice displayed slower gut transit time, alterations in ion transport, increased gut permeability, and enhanced fecal output in a novel environment stress paradigm. These alterations were accompanied by neuroanatomical changes in the ENS, including an indiscriminate loss of enteric neurons and region-specific loss of EGC. Spontaneous recovery of the microbiota restored the functional and morphological deficits, including motility and permeability alterations. Remarkably, spontaneous recolonization was also associated with the recovery of neurons, through enteric neurogenesis. Investigating which components of the microbiota were responsible for these effects, we found that neither LPS nor SCFA restored Abx-induced changes in gut function, though SCFA supplementation was able to reverse the stressinduced enhancement of fecal output. Despite not reversing most of the functional deficits, LPS was shown to enhance neuronal survival, and SCFA enhanced neuronal survival and promoted enteric neurogenesis. Why these significant changes in the structure of the ENS are not accompanied by functional recovery remains to be determined. Nevertheless, these findings suggest that other microbial components are critical for initiating the physiological function of new enteric neurons.

Reports in both germ-free and Abx-treated rodents have described alterations in baseline GI function, suggesting a role for the microbiota in the regulation of motility, ion transport, and permeability [21, 23, 24, 26, 4951]. While sex-dependent differences in the composition of the microbiota have been described [52], we observed similar physiological alterations in both sexes when bacteria were depleted by Abx treatment (e.g., dysmotility and increased gut permeability). These data suggest that, even though initial bacterial composition may be different, a core signaling mechanism is present in both microbiota, which, upon depletion, led to similar alterations in GI function in both sexes of mice. While previous work has shown that Abx exposure can directly influence neuronal function and intestinal motility patterns in vitro [53], others have described similar effects to intestinal physiology when analyzing both germ-free and Abx-treated mice, suggesting that the alterations in GI function are more likely to be related to the absence or depletion of the intestinal microbiota, rather than a side effect of the Abx treatment [18, 25, 54]. Additionally, to address whether the functional changes induced by depletion of the microbiota were permanent, we allowed mice to recover for 21 days after 14 days of Abx treatment (Fig. 5). Suez and colleagues reported that in mice, full restoration of the microbiota via spontaneous recolonization after Abx treatment can take longer than 21 days [55]. Nevertheless, we observed a significant normalization of GI function and overall microbial numbers following the 21-day recovery period. In agreement with our findings, germ-free and Abx-treated mice recolonized over a shorter period or colonized with reduced bacterial diversity (e.g., altered Schaedler flora), recovered specific GI functions, such as motility and intestinal permeability $[15,25,26]$. This suggests that the mechanism(s) driving these processes are likely not dependent on specific bacteria, but rather on common bacterial signaling pathways, such as those related to the MAMPs or abundant microbial secreted molecules. Therefore, we sought to test whether supplementation with either LPS or SCFA, both common microbe-derived mediators, could reverse or attenuate the changes induced by Abx treatment. These molecules have been associated with modulation of many different properties of the GI tract, including physiological (e.g., motility and intestinal permeability) and immune processes [34, 35, 47, 56, 57], and both have reduced concentrations after depletion of the microbiota $[18,58]$. Surprisingly, few beneficial effects on GI function were observed when Abx-treated mice were supplemented with either LPS or SCFA, with the only parameters normalized being the gut responses to a stress stimulus and the intestinal permeability. We showed that pellet output number after novel environment stress was recovered by SCFA supplementation, suggesting a potential role for SCFA in the modulation of the local response to stress, and long-term SCFA treatment restored the intestinal barrier. Thus, our results suggest a minor functional effect of LPS or SCFA supplementation, at least at the levels and intervals used in this study. This supports the notion of a more complex interplay between the host and the intestinal microbiota, likely involving multiple integrating mechanism(s) on the host to regulate GI physiology.

The ENS plays a fundamental role in regulating gut motility, secretion, and permeability [1, 59]. Previous studies have shown a modification of the ENS structure and function in germ-free rodents. These alterations consist of a reduction in neuronal density, including nitrergic neurons, and reduction in $\mathrm{EGC}$, in addition to altered electrophysiological responses in enteric neurons $[15,18,22,54,60]$. Our results support the hypothesis that depletion of bacteria induces enteric neuronal loss. However, alterations in EGC were not as widespread as the changes observed in neuronal populations. Previous reports have shown a reduction in EGC bodies in the ileal mucosa, but no clear alterations in the myenteric plexus [22], and reduction in the density of glial 
processes in the colonic myenteric plexus, but no changes in the number of EGC [61]. In contrast, we observed no changes in the number of $\mathrm{PLP}^{+}$EGC cell bodies in the mucosa after Abx treatment in both ileum and colon (Fig. 3), and we showed a reduction in both the number of EGC and the density of glial processes specifically in the ileal myenteric plexus. Exactly why there are differences between our results and the published literature is not clear and requires further investigation.

Interestingly, upon recolonization, ENS neuronal and glial densities were restored in both the ileum and the colon. These data suggest a correlation between a restored ENS structure with the recovery of function (Fig. 5). Moreover, enteric neuronal recovery has been recently demonstrated by others using a similar protocol of post-Abx treatment recolonization [25], in which the authors suggested a role for enteric postnatal neurogenesis mediated by the microbiota. Our findings are entirely consistent with these observations and extend them showing that not only do nestin-positive progenitor cells increase [25], but also Sox $2^{+}$cells that potentially arise from glial progenitor cells $[44,46,62]$. Enteric postnatal neurogenesis refers to the production of new neurons in the GI tract from birth to adulthood. The concept of neurogenesis in the brain of mammals in specific regions has now been widely accepted [63], however, this process within enteric neurons has been largely studied in vitro $[62,64,65]$. Only recently has enteric neurogenesis been demonstrated in an in vivo setting, with either healthy animals [43] or under conditions of inflammation or injury [44, 62, 64, 66]. It has become clear that the cell source for postnatal enteric neurogenesis is a glial cell or glial cell subtype [62, $64,67]$. The two glial markers most commonly used to demonstrate the glial-to-neuronal production of new neurons have been nestin $[25,43,65,68]$ and Sox2 $[44,46]$. Interestingly, these studies have focused on the myenteric plexus and, to date, no one has compared the submucosal and myenteric plexus in the same animals. Surprisingly, we observed differences in Sox 2 expression mediated by microbiota depletion when comparing the response to bacterial depletion in the two plexuses. These findings suggest that different mechanisms govern neurogenesis in the two plexuses in adult animals and raise many questions on how enteric neurogenesis is regulated and the extent to which the intestinal microbiota contributes to these processes. Nevertheless, the importance of this mechanism is underscored by the recent work from Matheis and colleagues, in which the authors showed that infection-induced neuronal loss can be reversed following reconstitution of the normal microbiota after Abx treatment [69].
One of the potential mechanisms by which the intestinal microbiota may modulate the ENS is via TLRs and their MAMP ligands [70, 71]. The expression of different TLRs in the ENS has been demonstrated previously [32, 33]. Anitha et al. elegantly showed the importance of neuronal TLR4 signaling in the control of gut motility [18], which was further confirmed by others [72]. Additionally, it has been shown that low-dose LPS has neuroprotective effects in both the central nervous system and ENS in vitro [18, 73]. In our experiments, while low-dose LPS supplementation did not reverse or attenuate the alterations in GI function induced by the Abx treatment, we observed a beneficial effect on neuronal survival when LPS was administered concomitantly with the Abx treatment. Our results complement previous in vitro reports $[18,73]$, demonstrating a role for the LPS-TLR4 signaling pathway in enteric neuronal survival. While LPS supplementation prevented Abxinduced neuronal loss, it is important to note that later supplementation with LPS after 14 days of Abx treatment did not rescue the neuronal loss. In vitro analysis has shown that LPS stimulates enteric neural progenitor cell proliferation, however, it slowed cell differentiation [74]. Thus, LPS-TLR4 signaling is likely associated with neuronal survival, rather than cell differentiation/neurogenesis. In addition to TLR4, TLR2 has been identified as a key regulator of the ENS and gut motility [19, 32]. A recent report described the effect of TLR2 in Abxinduced neuronal loss and altered gut functions [25]. The authors were able to reverse the Abx-induced neuronal loss by administering lipoteichoic acid (LTA), a TLR2 agonist, suggesting a role for TLR2 activation in neurogenesis after Abx-induced neuronal loss [25]. In hippocampal neurons, a dynamic complex modulation of both TLR2 and TLR4 has been implicated in neuronal survival and differentiation/neurogenesis [75]. TLR2 deficiency impairs the differentiation of neural progenitor cells into mature neurons, highlighting its importance in the neurogenesis process. Additionally, TLR4 seems to counteract and regulate TLR2 function in hippocampal neurons [75]. Based on our results, and recent literature, we speculate that in the ENS, TLR4 signaling is important for neuronal survival, while TLR2 activation may be required for neurogenesis. Nevertheless, additional studies are needed to delineate the complex interplay between TLR2 and TLR4 and their contribution to ENS neuronal survival and neurogenesis.

In addition to MAMPs, it has been shown that microbial-derived metabolites can modulate ENS and GI function. Reports have described a role for SCFA in increasing the expression of tryptophan hydroxylase 1 (Tph1; i.e., rate-limiting enzyme in the 5-HT synthesis pathway in enterochromaffin cells) in vitro [36]. Interestingly, dysmotility has been correlated with reductions in 
5-HT concentration in the gut in a reversible manner [20, 24, 36]. Furthermore, 5-HT has been associated with neurogenesis in the ENS, potentially via the $5-\mathrm{HT}_{4}$ receptor [45, 61]. Thus, we sought to test the effects of SCFA supplementation on GI function and ENS structure. While SCFA supplementation had little effect on the alterations in GI function induced by microbial depletion, we observed that this regimen led to a recovery of the enteric neurons after Abx treatment. These data suggest a role for SCFA in neuronal survival and/or neurogenesis after neuronal loss. We postulate that a potential mechanism by which SCFA may be acting on neurogenesis is via the $5-\mathrm{HT}_{4}$ receptor. Since SCFA increase Tph1 expression and levels of $5-\mathrm{HT}$ in the gut $[24,36]$, and the $5-\mathrm{HT}_{4}$ receptor has been implicated in neurogenesis $[45,61,76]$, it is plausible to hypothesize a link between the two. On the other hand, expression of free-fatty acid receptors, of which SCFA are agonists, has been demonstrated in enteric neurons [77]; however, their role in neuronal survival/proliferation/differentiation is still unknown. Thus, additional experiments are needed to determine the discrete mechanism(s) responsible for these beneficial effects of SCFA supplementation in our studies.

The implications of bacterial depletion in adult human health are still under investigation, but changes in barrier function appear to be critical. Here we show that a transient increase in gut permeability after Abx treatment can be normalized following bacterial recolonization in mice. However, we have not identified which region of the gut is responsible for this increase in permeability. Other reports have described the importance of the microbiota in regulating gut mucosal function in germ-free mice ex vivo, including secretion and maturation of the epithelial barrier [51, 78]. In accordance with our results, similar outcomes were described in Abx-treated adult mice, in which a transient increase in gut permeability was observed in vivo [26]. We assessed the role of LPS and SCFA in the modulation of the gut barrier after microbiota disruption. Previously, both molecules have been demonstrated to influence gut permeability [57, 79]. Even though no recovery effect was observed after intervention with LPS in our Abxtreated mice, long-term treatment with SCFA was able to reduce intestinal permeability. Interestingly, the presence of metronidazole in an Abx mix, or when administered alone, is associated with increased gut permeability, likely playing a crucial role in the effects observed $[26,80]$. Thus, future analysis of the bacteria being depleted or blooming after metronidazole treatment may shed light on potential microbe-driven mechanisms regulating intestinal permeability.

\section{Conclusions}

Our results suggest a role for the gut microbiota in constantly regulating a variety of GI functions in adulthood, independent of sex. Depletion of gut bacteria altered GI motility, secretion, and permeability. Moreover, the microbiota is essential for the maintenance of the ENS integrity, likely regulating enteric neuronal survival and promoting neurogenesis. MAMPs, such as LPS, may regulate enteric neuronal survival, while SCFA treatment was found to be associated with both survival and neurogenesis. Thus, we provide additional understanding of host-microbe interactions that contribute to the regulation of GI structure and function and the organization of the ENS.

\section{Methods \\ Animals}

Male and female $\mathrm{C} 57 \mathrm{Bl} / 6$ mice (Jackson Laboratories, Bar Harbor, ME, USA), 8-12 weeks old, were housed ( $n$ $=5 /$ cage $)$ in the animal facility at the University of Calgary at $22 \pm 2{ }^{\circ} \mathrm{C}$ on a 12-h light-dark cycle. Mice had free access to sterilized food and water. Upon arrival, mice were randomized, followed by a 1-week acclimation period before the start of any experiment. PLP1eGFP mice were developed by Dr. Wendy Macklin [40] and were bred in-house at the University of Colorado's animal facility. Male and female PLP1-eGFP littermate mice, 8-12 weeks old, were used in the experiments. Mice were kept under the same conditions as described above. All animal procedures were approved by the University of Calgary's Health Sciences Animal Care Committee (\#AC19-0124) and the University of Colorado's Anschutz Institutional Animal Care and Use Committee (\#00088), in accordance with the guidelines established by the Canadian Council on Animal Care and the PHS Policy on Humane Care and Use of Laboratory Animals, respectively.

\section{Treatments \\ Broad-spectrum antibiotic treatment}

Abx treatment was administered according to previously published protocols [54, 56]. Broad-spectrum Abx mix was diluted in sterilized water and consisted of ampicillin (1 g/L, A9518; Sigma-Aldrich, St. Louis, MO, USA), neomycin (1 g/L, N1876; Sigma-Aldrich), vancomycin (0.5 g/L, 94747; Sigma-Aldrich), and metronidazole ( $1 \mathrm{~g} /$ L, M3761; Sigma-Aldrich). Mice received the Abx solution for at least 14 days. Due to the body weight loss observed at the beginning of the Abx treatment in a pilot experiment (Figure S8), also shown by others [81], we opted to gradually introduce metronidazole in the $\mathrm{Abx}$ solution as previously described [38]. Briefly, no metronidazole was added on day $0 ; 0.25 \mathrm{~g} / \mathrm{L}$ ( $25 \%$ of final concentration) was added at day $2 ; 0.50 \mathrm{~g} / \mathrm{L}(50 \%$ of final 
concentration) was added at day 6 ; and $1.0 \mathrm{~g} / \mathrm{L}$ (full concentration) was added at day 9. Abx solution was refilled every week. Body weight was assessed three times a week to monitor body weight changes. To assess the effects of spontaneous microbiota recolonization, bottles of Abx were swapped for regular autoclaved water at day 14 . Mice had access to water for 21 days after the bottle swap to allow for microbiota recolonization.

\section{Low-dose LPS supplementation}

Low-dose LPS supplementation was administered as described previously $[38,56]$. LPS (Escherichia coli O111: B4; L2630, Sigma-Aldrich) was diluted $(50 \mu \mathrm{g} / \mathrm{ml})$ in the Abx solution. Animals had free access to the Abx solution containing LPS from day 0 , or after 14 days of Abxtreatment only, depending on the treatment group. Mice received LPS supplementation for 21 or 7 days prior to any experiment. LPS concentration was chosen based on promising beneficial effects on gut motility [47].

\section{SCFA mix supplementation}

A mixture of SCFA was administered according to previous reports [48, 82, 83]. SCFA mixture was composed of $67.5 \mathrm{mM}$ of acetate (S2889, Sigma-Aldrich), $25.9 \mathrm{mM}$ of propionate (P1880, Sigma-Aldrich), and $40 \mathrm{mM}$ of butyrate (303410, Sigma-Aldrich). The mixture was diluted in the Abx solution. Solutions with no SCFA mixture were sodium matched. All solutions were $\mathrm{pH}$ controlled (7.4-7.6) and changed weekly.

\section{In vivo measurements}

Novel environment stress response and fecal water content Mice were placed individually in new bedding-free cages and monitored for $1 \mathrm{~h}$. Fecal pellets were collected and weighed every $15 \mathrm{~min}$. Feces were dried at $50{ }^{\circ} \mathrm{C}$ for 24 $\mathrm{h}$ and re-weighed. Wet and dry weights were used to generate the fecal pellet wet:dry ratio.

\section{Whole gut transit time}

The assessment of the whole gut transit time was performed immediately following the novel environment stress test. Mice were gavaged with $200 \mu \mathrm{L}$ of the nonabsorbable dye Evans Blue (5\% suspended in 5\% Gum Arabic; Sigma-Aldrich) and the period from the time of the gavage to the appearance of the first blue-colored fecal pellet was considered the whole gut transit time. Tests were normally ended at $480 \mathrm{~min}$. However, if $20 \%$ or more of the animals being tested had not expelled a blue pellet by $480 \mathrm{~min}$, they were allowed an extra 120 min of testing, with the test ending at $600 \mathrm{~min}$.

\section{Assessment of small intestine transit}

Mice were gavaged with $200 \mu \mathrm{L}$ of the non-absorbable dye Evans Blue (5\% suspended in 5\% Gum Arabic), and
$15 \mathrm{~min}$ later, they were euthanized under isofluorane anesthesia. The small intestine was immediately removed and measured (pyloric sphincter to ileal-cecal junction). Distance traveled by the dye was also measured. Results are expressed as a percentage of the length covered by the dye over the total small intestinal length. This test was performed concomitantly with the intestinal permeability assay such that mice received the Evans Blue gavage $3 \mathrm{~h} 45 \mathrm{~min}$ following the FSA gavage for the intestinal permeability assay (see below).

\section{Distal colonic transit time}

We used the bead expulsion test to assess distal colonic propulsion. Mice were lightly anesthetized with isoflurane and a $2.5-\mathrm{mm}$ spherical plastic bead coated in nail varnish was gently inserted $2 \mathrm{~cm}$ into the distal colon using a silicone pusher. Mice were placed in individual, bedding-free cages, and latency time for bead expulsion was recorded. After bead expulsion, mice were returned to their home cage. Experiments were repeated 3 times with $100 \mathrm{~min}$ intervals. The mean of 3 experiments was considered the time for bead expulsion.

\section{Intestinal permeability assay}

Mice were gavaged with $100 \mu \mathrm{L}$ of $50 \mathrm{mg} / \mathrm{ml}$ FSA (478 daltons; Setareh Biotech, Eugene, OR, USA). After $4 \mathrm{~h}$, mice were anesthetized under isofluorane. Blood was drawn by cardiac puncture and collected in a Microtainer SST Tube (BD Company, Franklin Lakes, NJ, USA) after which mice were euthanized and the small intestine removed to determine small intestinal transit as above. At room temperature, blood was allowed to clot for at least $30 \mathrm{~min}$, followed by centrifugation at $2000 \times g$ for $10 \mathrm{~min}$. The supernatant (serum) was collected, pipetted $(50 \mu \mathrm{L})$ in triplicate into a 96-well plate, and read at $485 / 535 \mathrm{~nm}$ in a spectrophotometer. Sample FSA concentration $(\mu \mathrm{g} / \mathrm{mL}$ of serum) was determined using a standard curve.

\section{Tissue harvesting}

After in vivo experiments, mice were either anesthetized with isoflurane and euthanized by cervical dislocation, or euthanized by $\mathrm{CO}_{2}$ asphyxiation followed by cervical dislocation. The entire gut was removed, and different regions were allocated for different analyses. The cecal contents were removed and the cecum was weighed. The length of the small intestine and colon was measured. The distal ileum was divided for immunofluorescence and Ussing chamber tests. The proximal colon was harvested for immunofluorescence.

\section{Immunofluorescence labeling}

Dissection of submucosal and myenteric plexuses was done similarly in both the ileum and colon. To obtain 
whole-mount preparations, samples were first bathed in a solution containing $1 \mu \mathrm{M}$ nifedipine (Sigma-Aldrich) diluted in PBS for $10 \mathrm{~min}$. Next, tissues were opened along the mesenteric border and pinned out in a petri dish containing Sylgard coating with the serosal side facing down. Samples were fixed with either Zamboni's fixative for $24 \mathrm{~h}$ at $4{ }^{\circ} \mathrm{C}$, or specific for ChAT staining and PLP1-eGFP mice, with $4 \%$ paraformaldehyde (ThermoFisher Scientific, Waltham, MA, USA) for $2 \mathrm{~h}$ at $4{ }^{\circ} \mathrm{C}$. Tissues were then washed with PBS containing 5\% sodium azide (3 times $10 \mathrm{~min}$ each) and stored at $4{ }^{\circ} \mathrm{C}$ until further processing. To obtain submucosal and myenteric preparations, samples were dissected under a microscope; submucosal preparations were obtained by gently scraping the mucosa away and peeling off the submucosal plexus, and the myenteric preparations were acquired by stripping off the mucosa/submucosa layers and the circular muscle leaving a preparation consisting of the longitudinal muscle and associated myenteric plexus. Following the acquisition of submucosal and myenteric preparations, samples were processed for immunohistochemistry. First, samples were incubated with primary antibody (Table 1) for $48 \mathrm{~h}$ at $4{ }^{\circ} \mathrm{C}$. The primary antibody solution was removed, samples were washed (3 times 5 min each) with PBS containing 0.1\% Triton X100 (Sigma-Aldrich), and incubated with secondary antibody (Table 1 ) for $1-2 \mathrm{~h}$ at room temperature followed by washing ( 3 times 5 min each) with PBS. When double labeling, samples were washed with PBS (3 times of 10 min each), and the second labeling was performed following the same protocol. Particularly when using antiSox2 antibodies, samples were pre-treated with DMSO (VWR International, Edmonton, $\mathrm{AB}$, Canada) for $30 \mathrm{~min}$ at room temperature prior to primary antibody incubation. Whole-mount preparations were mounted with bicarbonate-buffered glycerol on microscope slides, stored at $4{ }^{\circ} \mathrm{C}$ under dark conditions for further analyses. All antibodies used were diluted in a PBS solution containing $0.1 \%$ Triton $\mathrm{X}-100,10 \%$ bovine serum albumin (BSA), 5\% sodium azide, 4\% EDTA. Tissues from PLP1eGFP mice were counterstained with DAPI (InvitrogenThermoFisher Scientific, Waltham, MA, USA) and mounted with Prolong Gold (Invitrogen-ThermoFisher Scientific).

To obtain cryosections from PLP1-eGFP mice, distal ileum and colon were harvested and fixed in $4 \%$ paraformaldehyde (ThermoFisher Scientific) diluted in PBS for $24 \mathrm{~h}$ at $4{ }^{\circ} \mathrm{C}$. After rinsing with PBS, tissues were submerged in $20 \%$ sucrose in PBS for $24 \mathrm{~h}$ at $4{ }^{\circ} \mathrm{C}$ and embedded in O.C.T. compound (ThermoFisher Scientific) on a bed of dry ice. Cryosections were cut at $20 \mu \mathrm{m}$ and collected on poly-D-lysine (Sigma-Aldrich) coated slides, with intervals of $40-80 \mu \mathrm{m}$ between each collected section. Cryosections were equilibrated to room

Table 1 Antibody reagent list

\begin{tabular}{|c|c|c|c|}
\hline Antibodies & Dilution & Source & Identifier \\
\hline Mouse anti-HuC/D & $1: 200$ & Invitrogen & Cat\#: A21271; RRID: AB_221448 \\
\hline Donkey anti-mouse Alexa Fluor 488 & $1: 200$ & Jackson ImmunoResearch & Cat\#: 715-546-151; RRID: AB_2340850 \\
\hline Sheep anti-nNOS & $1: 200$ & Millipore Sigma & Cat\#: AB1529; RRID: AB_90743 \\
\hline Donkey anti-sheep Cy3 & $1: 100$ & Jackson ImmunoResearch & Cat\#: 713-165-147; RRID: AB_2315778 \\
\hline Goat anti-ChAT & $1: 50$ & Millipore Sigma & Cat\#: AB144P; RRID: AB_90661 \\
\hline Donkey anti-goat Cy3 & $1: 200$ & Jackson ImmunoResearch & Cat\#: 705-165-147; RRID: AB_2307351 \\
\hline Rabbit anti-S100B & $1: 500$ & Agilent & Cat\#: GA50461-2; RRID: AB_10013383 \\
\hline Donkey anti-rabbit Cy3 & $1: 100$ & Jackson ImmunoResearch & Cat\#: 711-165-152; RRID: AB_2307443 \\
\hline Goat anti-Sox2 & $1: 200$ & R\&D Systems & Cat\#: AF2018; RRID: AB_355110 \\
\hline Donkey anti-goat Cy3 & $1: 200$ & Jackson ImmunoResearch & Cat\#: 705-165-147; RRID: AB_2307351 \\
\hline Rabbit anti-S100 & $1: 200$ & ThermoFisher Scientific & Cat\#: RB-044-AO; RRID: AB_60518 \\
\hline Donkey anti-rabbit Alexa Fluor 647 & $1: 400$ & Invitrogen & Cat\#: A-31573; RRID: AB_2536183 \\
\hline Mouse anti-Tuj1 & $1: 200$ & Biolegend & Cat\#: 801201; RRID: AB_ 2313773 \\
\hline Donkey anti-mouse Alexa Fluor 488 & $1: 200$ & Jackson ImmunoResearch & Cat\#: 715-546-151; RRID: AB_2340850 \\
\hline Goat anti-calretinin & $1: 200$ & Swant & Cat\#: CG1; RRID: AB_10000342 \\
\hline Donkey anti-goat Cy3 & $1: 200$ & Jackson ImmunoResearch & Cat\#: 705-165-147; RRID: AB_2307351 \\
\hline Rat anti-Ki67 & $1: 400$ & Invitrogen & Cat\#: 14-5698-82; RRID: AB_10854564 \\
\hline Goat anti-rat Alexa Fluor 647 & $1: 400$ & Invitrogen & Cat\#: A21247; RRID: AB_141778 \\
\hline Human anti-Hu & $1: 10,000$ & Mayo Clinic & Cat\#: N/A; RRID: AB_2314657 \\
\hline Goat anti-human Alexa Fluor 647 & $1: 400$ & Invitrogen & Cat\#: A21445; RRID: AB_2535862 \\
\hline
\end{tabular}


temperature, fixed in $4 \%$ paraformaldehyde for $20 \mathrm{~min}$, and blocked in 10\% donkey serum, 10\% BSA and 1\% Triton X-100 diluted in PBS for $1 \mathrm{~h}$ at room temperature. Here, a rabbit polyclonal antibody for S100 (ThermoFisher Scientific) at 1:200 diluted in blocking buffer was applied to the sections overnight at $4{ }^{\circ} \mathrm{C}$. Sections were washed with PBS and incubated in donkey anti-rabbit secondary antibody conjugated to Alexa Fluor-647 (Invitrogen) at 1:400 diluted in blocking buffer for $1 \mathrm{~h}$ at room temperature. For Ki67 immunolabeling, a similar protocol was used, applying Ki67 monoclonal antibody (ThermoFisher Scientific) and $\mathrm{HuC} / \mathrm{D}$ (Mayo Clinic). Sections were washed with PBS, counterstained with DAPI (Invitrogen), and coverslipped with Prolong Gold (Invitrogen).

\section{Quantification of enteric neurons and EGC}

Analyses for neuronal $\left(\mathrm{HuC} / \mathrm{D}^{+}, \mathrm{nNOS}^{+}, \mathrm{ChAT}^{+}\right.$, Calretinin $^{+}$, Sox $2^{+}$) counts and $\mathrm{S}_{100 \mathrm{~B}^{+}}$EGC were done using a Zeiss Axioplan fluorescence microscope (Zeiss Canada, Toronto, ON, Canada). Analyses of PLP1-eGFP mouse samples were undertaken on an Olympus IX83 motorized inverted microscope (Olympus, Center Valley, PA, USA). For neuronal counts, 10 ganglia were randomly selected and immunolabelled cells were quantified, within each preparation, either submucosal or myenteric. Data for each animal is composed of the mean of the 10 ganglia counted. For Tuj $1^{+}$neuronal fiber analysis, the first images were captured using the Zeiss Axioplan fluorescence microscope (Zeiss Canada) applying the same exposure time to all samples. By using the FIJI software (ImageJ, NIH, Bethesda, MD, USA) for image analysis, the ganglia were delineated, and the fluorescence intensity measured. Background fluorescence intensity was subtracted to get a corrected fluorescence intensity. Ten ganglia were measured per region, and averaged for the final value. For $\mathrm{S} 100 \mathrm{~B}^{+} \mathrm{EGC}$ numbers, a total ganglionic area of $0.020-0.036 \mu \mathrm{m}^{2}$ for submucosal and $0.20-0.23 \mu \mathrm{m}^{2}$ for myenteric was analyzed per mouse, and $\mathrm{S} 100 \mathrm{~B}^{+}$cells were counted within this area. Data are presented as normalized to control values due to variation in tissue stretching during fixation among cohorts. Mucosal $\mathrm{PLP}^{+}{ }^{+}$EGC were manually quantified in villi and crypts along the entire circumference of the ileum. An average of 35 intact villus-crypt units were counted per mouse. For colonic analysis, an average of $1.5 \mathrm{~mm}^{2}$ of mucosal area was analyzed per mouse. Percent area measurements of the PLP1 ${ }^{+}$signal in images of whole-mount preparations were analyzed with FIJI (ImageJ, NIH) to determine glial cell density. An average of 4 images were analyzed per animal. All quantification was done by an experimenter blinded to the treatments.

\section{In vivo cell proliferation assay}

Mice received $100 \mu \mathrm{L}$ of $10 \mathrm{mg} / \mathrm{mL}$ of EdU (ThermoFisher Scientific) diluted in molecular grade water by intraperitoneal injection 7 days following the start of $\mathrm{Abx}$ treatment or $1 \mathrm{mg}$ of EdU per mouse. Click-IT Plus EdU Alexa Fluor 594 Imaging Kit (Invitrogen) was used according to manufacturer instructions to visualize the EdU in cryosections of the distal ileum and colon of the PLP1-eGFP mouse.

\section{Measurement of ion transport and transepithelial electrical resistance}

Immediately after euthanasia, full-thickness segments of terminal ileum were removed, opened along the mesenteric border, cleaned of luminal contents, and mounted in Ussing chambers (Physiologic Instruments, San Diego, CA, USA) with an exposed area of $0.3 \mathrm{~cm}^{2}$. The tissues were bathed at $37{ }^{\circ} \mathrm{C}$ in oxygenated $\left(95 \% \mathrm{O}_{2}-5 \% \mathrm{CO}_{2}\right)$ Krebs solution ( $\mathrm{pH} 7.4)$ with $10 \mathrm{mM}$ of glucose and mannitol in the serosal and mucosal compartments, respectively. Tissues were held under voltage-clamp conditions $(0 \mathrm{~V})$ and allowed to equilibrate for $20 \mathrm{~min}$. Net electrogenic ion transport across the epithelium was recorded as short-circuit current (Isc; $\mu \mathrm{A} / \mathrm{cm}^{2}$ ). The measurement of transepithelial potential and Isc allowed calculation of transepithelial electrical resistance (TER; $\Omega / \mathrm{cm}^{2}$ ) according to Ohm's law. Changes in net electrogenic ion flux were evaluated by measuring changes in Isc in response to neuronal depolarization with veratridine $(10 \mu \mathrm{M}$ serosal side; Calbiochem, 676950, San Diego, CA, USA) or in response to muscarinic receptor stimulation with carbachol $(100 \mu \mathrm{M}$ serosal side; C4382, Sigma-Aldrich). The difference between basal Isc and peak Isc recorded after veratridine or carbachol addition was measured $\left(\Delta \mathrm{Isc} ; \mu \mathrm{A} / \mathrm{cm}^{2}\right)$. A positive $\Delta$ Isc indicated a luminally directed negative net charge transfer (anion secretion). Measurements were conducted and averaged in two adjacent ileal segments from the same mouse.

\section{Small intestine relaxation assay}

The small intestine was removed and measured. The tissue was then placed in a $1 \mu \mathrm{M}$ nifedipine solution for 10 min, after which the length of the small intestine was measured again and the variation (\%) in length was determined.

\section{Quantification of fecal bacterial load}

Fecal pellets (1-3) were collected in a sterile tube and fixed immediately on dry ice. Samples were stored at $80{ }^{\circ} \mathrm{C}$ until analysis. DNA was extracted using a DNeasy PowerSoil kit (Qiagen, Toronto, ON, Canada) according to the manufacturer's instructions, with minor modifications. Briefly, sample homogenization was done applying $30 \mathrm{~Hz}$ for $1 \mathrm{~min}$ in the TissueLyzer LT (Qiagen) and 
eluted in nuclease-free water. DNA load was quantified using NanoDrop (ThermoFisher Scientific) and standardized to $1 \mathrm{ng} / \mu \mathrm{L}$ in all samples. Next, a qPCR reaction was carried out using PerfeCTa SYBR Green SuperMix (QuantaBio, Beverly, MA, USA). Universal bacterial primers UniF340 and UniR514 [84] were used to amplify bacterial DNA. The qPCR mixture solution consisted of $10 \mu \mathrm{L}$ reactions containing: $5 \mu \mathrm{L}$ of master mix, $0.5 \mu \mathrm{L}$ of $5 \mathrm{mM}$ UniF340, $0.5 \mu \mathrm{L}$ of $5 \mathrm{mM}$ UniR514, $2 \mu \mathrm{L}$ of nuclease-free water, and $2 \mu \mathrm{L}$ of sample. The qPCR thermocycler (StepOnePlus; Applied Biosystems, Foster City, CA, USA) was set for an initial step at $95{ }^{\circ} \mathrm{C}$ for $3 \mathrm{~min}$, followed by 40 cycles of $10 \mathrm{~s}$ at $95{ }^{\circ} \mathrm{C}$ and 45 $\mathrm{s}$ at $63{ }^{\circ} \mathrm{C}$. Data were acquired in the final step at $63{ }^{\circ} \mathrm{C}$. A melting curve was acquired at the end of the run. Results were calculated based on a standard curve generated with bacterial genomic DNA from a pure bacterium colony of Clostridium sporogeneses.

\section{Statistical analysis}

Data is presented as mean \pm standard error of the mean (SEM). Shapiro-Wilk's and Kolmogorov-Smirnov's tests were used to assess data normality. Student's $t$ test was applied for analysis between two groups. For multiple comparisons, we used one-way ANOVA, followed by Tukey's multiple comparison test. Analyses of two variables were done with Two-way ANOVA, followed by either Tukey's or Sidak's multiple comparison test. The specific statistical test used in each figure panel is described in the figure legends. $n$ is variable among experiments and described in every figure legend. Bar graphs display biological replicates as individual dots. $p<0.05$ was accepted as statistically significant. Statistical analyses were performed with GraphPad Prism 8 (GraphPad Software, La Jolla, CA, USA).

\section{Abbreviations}

5-HT: Serotonin; Abx: Antibiotic; EGC: Enteric glial cell; ENS: Enteric nervous system; FSA: Fluorescein-5-6-sulfonic acid; Gl: Gastrointestinal; LPS: Lipopolysaccharide; MAMPs: Microbial-associated molecular patterns; SCFA: Short-chain fatty acid; TER: Transepithelial electrical resistance; TLR: Toll-like receptor

\section{Supplementary Information}

The online version contains supplementary material available at https://doi. org/10.1186/s40168-021-01165-z.

Additional file 1: Figure S1. Variation in small intestine length after muscle relaxant treatment ex vivo. Small intestine was removed from male mice and placed in a solution containing nifedipine $(1 \mu \mathrm{M})$ for 10 minutes. (a) Small intestinal length variation before and after nifedipine treatment. ${ }^{* *} p<0.01,{ }^{* * *} p<0.001$, Student's $t$ test comparing Control vs Abx. (b) Small intestine length relative variation after nifedipine; $p>0.05$, Student's $t$ test. Data in panel $b$ are expressed as mean \pm SEM. $N=4-5$.

Additional file 2: Figure S2. Antibiotic (Abx) treatment induces a reduction in the number of enteric neurons. Representative immunofluorescent images of ganglia in the submucosal and myenteric plexuses in the colon: (a) HuC/D+ (green) and $\mathrm{nNOS}^{+}$(magenta) neurons; (b) $\mathrm{HuC/D}{ }^{+}$(green) and $\mathrm{ChAT}^{+}$(magenta) neurons. Scale bar: $30 \mu \mathrm{m}$.

Additional file 3: Figure S3. Reduction in $\mathrm{Tuj}^{+}{ }^{+}$neuronal fibers and $\mathrm{CALR}^{+}$neurons is observed after depletion of gut bacteria (a) Representative immunofluorescent images of ganglia in the submucosal and myenteric plexuses: Tuj ${ }^{+}$(green) neuronal fibers in the ileum and colon of control and antibiotic (Abx)-treated mice. Scale bar: $30 \mu \mathrm{m}$. (b) Quantification of Tuj $1^{+}$neuronal fibers in both ileal and colonic submucosal and myenteric plexuses. (c) Representative immunofluorescent images of ganglia in the submucosal and myenteric plexuses: calretinin $(C A L R)^{+}$(magenta) neurons in the ileum and colon of control and antibiotic (Abx)-treated mice. Scale bar: $30 \mu \mathrm{m}$. (d) Number of CALR ${ }^{+}$neurons in both ileal and colonic submucosal and myenteric plexuses. Data are expressed as mean \pm SEM. $n=3-5 .{ }^{* *} p<0.01,{ }^{* * *} p<$ 0.001 ; Student's $t$ test.

Additional file 4: Figure S4. Overlapping of PLP1 and S100B expression in enteric glial cells. Representative images of immunofluorescence in the colon of PLP1-eGFP mice stained with antiS100B (white) antibody. Scale bar: $100 \mu \mathrm{m}$.

Additional file 5: Figure S5. Proliferative markers are not present in the ENS of either control or antibiotic (Abx)-treated mice. (a) Representative immunofluorescent images of sections of the ileum and the colon: $\mathrm{HuCl}$ $\mathrm{D}^{+}$neurons (red), $\mathrm{PLP1}^{+}$glia (green), Ki67 ${ }^{+}$proliferating cells (gray), merge with DAPI (blue) background staining. Scale bar: $50 \mu \mathrm{m}$. (b) Representative immunofluorescent images of sections of the ileum and the colon: HuC/D ${ }^{+}$neurons (red), $\mathrm{PLP}^{+}$glia (green), EdU ${ }^{+}$proliferating cells (gray), merge with DAPI (blue) background staining. Scale bar: 50 $\mu \mathrm{m}$.

Additional file 6: Figure S6. Fecal bacterial load returns to control levels after antibiotic (Abx) withdrawal. Data are expressed as mean \pm SEM. $n=3-5$. n.d.: not detectable.

Additional file 7: Figure S7. Spontaneous microbiota recolonization restores antibiotic (Abx)-induced enteric neuronal loss.

Immunofluorescent images of representative ganglia of the submucosal and myenteric plexuses in the colon: $\mathrm{HuC}^{+} \mathrm{D}^{+}$(green) and $\mathrm{nNOS}^{+}$

(magenta) neurons. Scale bar: $30 \mu \mathrm{m}$.

Additional file 8: Figure S8. Administration of the full concentration of metronidazole at the start of the experiment leads to body weight loss. Adult male mice were treated with a combination of antibiotics for 14 days in the drinking water (Abx group). The antibiotic regimen consisted of ampicillin (1 $\mathrm{g} / \mathrm{L})$, neomycin $(1 \mathrm{~g} / \mathrm{L})$, vancomycin $(0.5 \mathrm{~g} / \mathrm{L})$, and metronidazole $(1 \mathrm{~g} / \mathrm{L})$. Graph shows body weight variation over the course of the 14-day experiment. Data are expressed as mean \pm SEM. $n=$ 8. ${ }^{* * *} p<0.001$; two-way ANOVA, followed by Sidak's multiple comparison test.

\section{Acknowledgements}

The authors thank Lateece Griffin, Holly Szczepanski, and Sangkha Paul for their outstanding technical support. We also thank Dr. Matthew Stephens for helping with LPS experiments and Erik van Tilburg Bernardes for the support with bacterial quantification in the feces. We thank Dr. Wallace MacNaughton and Dr. Cristiane Baggio for support with Ussing chamber experiments. SAH holds the Canada Research Chair (Tier 2) in Host-microbe Interactions and Chronic Disease and the Dr. Lloyd Sutherland Investigatorship in Inflammatory Bowel Disease/Gl Research, at the University of Calgary.

\section{Authors' contributions}

FAV, CMK, J-BC, JB-G, SAH, and KAS designed the studies; FAV, CMK, LEW, $C W, J-B C$, and ARF conducted the experiments and performed data analyses; FAV, SAH, and KAS drafted the manuscript. All authors had access to the study data and had critically reviewed and approved the final manuscript for submission. FAV, JB-C, WBM, JB-G, SAH, and KAS obtained funding for the study.

\section{Funding}

This work was supported by grants from the Canadian Institutes of Health Research (SAH, KAS), and a Canadian Institutes of Health Research Team Grant: Health Challenges in Chronic Inflammation (SAH, KAS; Principal 
Investigator: Dr. Mark G. Swain), Department of Pediatrics and Digestive Health Institute - University of Colorado/Children's Hospital Colorado (JB-G) and National Institutes of Health (WBM). FAV was supported by the National Council for Scientific and Technological Development, Brazil. J-BC was supported by the Human Frontier Science Program and Alberta Innovates.

\section{Availability of data and materials}

All data needed to evaluate the conclusions in the paper are present in the paper. Additional data related to this paper may be requested from the authors.

\section{Declarations}

\section{Ethics approval and consent to participate}

All animal procedures were approved by the University of Calgary's Health Sciences Animal Care Committee (\#AC19-0124) and the University of Colorado's Anschutz Institutional Animal Care and Use Committee (\#00088), in accordance with the guidelines established by the Canadian Council on Animal Care and the PHS Policy on Humane Care and Use of Laboratory Animals, respectively.

\section{Consent for publication}

Not applicable.

\section{Competing interests}

The authors declare no conflicts of interest.

\section{Author details}

${ }^{1}$ Hotchkiss Brain Institute, University of Calgary, Calgary, AB T2N 4N1, Canada. ${ }^{2}$ Snyder Institute for Chronic Diseases, University of Calgary, Calgary, AB T2N 4N1, Canada. ${ }^{3}$ Inflammation Research Network, University of Calgary, Calgary, AB T2N 4N1, Canada. ${ }^{4}$ Department of Physiology and Pharmacology, Cumming School of Medicine, University of Calgary, 3330 Hospital Drive NW, Calgary, AB T2N 4N1, Canada. ${ }^{5}$ Department of Pediatrics, Section of Gastroenterology, Hepatology and Nutrition, University of Colorado, Aurora, CO 80045, USA. ${ }^{6}$ Department of Cell and Developmental Biology, University of Colorado School of Medicine, Aurora, CO 80045, USA.

${ }^{7}$ Neurogastroenterology and Motility Program, Digestive Health Institute, Children's Hospital Colorado, Aurora, CO 80045, USA. ${ }^{8}$ Alberta Children's Hospital Research Institute, University of Calgary, Calgary, AB T2N 4N1, Canada.

\section{Received: 10 August 2021 Accepted: 15 September 2021}

\section{Published online: 26 October 2021}

\section{References}

1. Furness JB. The enteric nervous system and neurogastroenterology. Nat Rev Gastroenterol Hepatol. 2012;9(5):286-94. https://doi.org/10.1038/nrgastro.2 012.32.

2. Sharkey KA, Beck PL, McKay DM. Neuroimmunophysiology of the gut: advances and emerging concepts focusing on the epithelium. Nat Rev Gastroenterol Hepatol. 2018;15(12):765-84. https://doi.org/10.1038/s41575018-0051-4

3. Gulbransen BD, Sharkey KA. Novel functional roles for enteric glia in the gastrointestinal tract. Nat Rev Gastroenterol Hepatol. 2012;9(11):625-32. https://doi.org/10.1038/nrgastro.2012.138.

4. Spencer NJ, Hu H. Enteric nervous system: sensory transduction, neural circuits and gastrointestinal motility. Nat Rev Gastroenterol Hepatol. 2020; 17(6):338-51. https://doi.org/10.1038/s41575-020-0271-2.

5. Yoo BB, Mazmanian SK. The enteric network: interactions between the immune and nervous systems of the gut. Immunity. 2017:46(6):910-26. https://doi.org/10.1016/j.immuni.2017.05.011.

6. Brierley SM, Linden DR. Neuroplasticity and dysfunction after gastrointestinal inflammation. Nat Rev Gastroenterol Hepatol. 2014;11(10):611-27. https:// doi.org/10.1038/nrgastro.2014.103.

7. Black CJ, Ford AC. Global burden of irritable bowel syndrome: trends, predictions and risk factors. Nat Rev Gastroenterol Hepatol. 2020;17(8):47386. https://doi.org/10.1038/s41575-020-0286-8.

8. Collaborators GIBD. The global, regional, and national burden of inflammatory bowel disease in 195 countries and territories, 1990-2017: a systematic analysis for the Global Burden of Disease Study 2017. Lancet Gastroenterol Hepatol. 2020;5:17-30.

9. Ni J, Wu GD, Albenberg L, Tomov VT. Gut microbiota and IBD: causation or correlation? Nat Rev Gastroenterol Hepatol. 2017;14(10):573-84. https://doi. org/10.1038/nrgastro.2017.88.

10. Chang $\mathrm{C}$, Lin H. Dysbiosis in gastrointestinal disorders. Best Pract Res Clin Gastroenterol. 2016;30(1):3-15. https://doi.org/10.1016/j.bpg.2016.02.001.

11. Collins SM. A role for the gut microbiota in IBS. Nat Rev Gastroenterol Hepatol. 2014;11(8):497-505. https://doi.org/10.1038/nrgastro.2014.40.

12. Hung LY, Boonma $P$, Unterweger $P$, Parathan $P$, Haag $A$, Luna RA, et al. Neonatal antibiotics disrupt motility and enteric neural circuits in mouse colon. Cell Mol Gastroenterol Hepatol. 2019;8:298-300.e296.

13. O'Mahony SM, Felice VD, Nally K, Savignac HM, Claesson MJ, Scully P, et al. Disturbance of the gut microbiota in early-life selectively affects visceral pain in adulthood without impacting cognitive or anxiety-related behaviors in male rats. Neuroscience. 2014;277:885-901. https://doi.org/10.1016/j. neuroscience.2014.07.054.

14. Cho I, Yamanishi S, Cox L, Methé BA, Zavadil J, Li K, et al. Antibiotics in early life alter the murine colonic microbiome and adiposity. Nature. 2012; 488(7413):621-6. https://doi.org/10.1038/nature11400.

15. Collins J, Borojevic R, Verdu EF, Huizinga JD, Ratcliffe EM. Intestinal microbiota influence the early postnatal development of the enteric nervous system. Neurogastroenterol Motil. 2014;26(1):98-107. https://doi. org/10.1111/nmo.12236.

16. Mao YK, Kasper DL, Wang B, Forsythe P, Bienenstock J, Kunze WA Bacteroides fragilis polysaccharide $\mathrm{A}$ is necessary and sufficient for acute activation of intestinal sensory neurons. Nat Commun. 2013;4(1):1465. https://doi.org/10.1038/ncomms2478.

17. Hung LY, Parathan $P$, Boonma $P$, Wu Q, Wang Y, Haag A, et al. Antibiotic exposure postweaning disrupts the neurochemistry and function of enteric neurons mediating colonic motor activity. Am J Physiol Gastrointest Liver Physiol. 2020;318(6):G1042-g1053. https://doi.org/10.1152/ajpgi.00088.2020.

18. Anitha M, Vijay-Kumar M, Sitaraman SV, Gewirtz AT, Srinivasan S. Gut microbial products regulate murine gastrointestinal motility via Toll-like receptor 4 signaling. Gastroenterology. 2012;143:1006-1016.e1004.

19. Caputi V, Marsilio I, Filpa V, Cerantola S, Orso G, Bistoletti M, et al. Antibioticinduced dysbiosis of the microbiota impairs gut neuromuscular function in juvenile mice. Br J Pharmacol. 2017;174(20):3623-39. https://doi.org/10.1111/ bph.13965.

20. Ge X, Ding C, Zhao W, Xu L, Tian H, Gong J, et al. Antibiotics-induced depletion of mice microbiota induces changes in host serotonin biosynthesis and intestinal motility. J Transl Med. 2017;15(1):13. https://doi. org/10.1186/s12967-016-1105-4

21. Grasa L, Abecia L, Forcén R, Castro M, de Jalón JAG, Latorre E, et al. Antibiotic-induced depletion of murine microbiota induces mild inflammation and changes in Toll-like receptor patterns and intestinal motility. Microb Ecol. 2015;70(3):835-48. https://doi.org/10.1007/s00248-0150613-8.

22. Kabouridis PS, Lasrado R, McCallum S, Chng SH, Snippert HJ, Clevers H, et al. Microbiota controls the homeostasis of glial cells in the gut lamina propria. Neuron. 2015;85(2):289-95. https://doi.org/10.1016/j.neuron.2014.12.037.

23. Obata Y, Castaño Á, Boeing S, Bon-Frauches AC, Fung C, Fallesen T, et al. Neuronal programming by microbiota regulates intestinal physiology. Nature. 2020;578(7794):284-9. https://doi.org/10.1038/s41586-020-1975-8.

24. Yano JM, Yu K, Donaldson GP, Shastri GG, Ann P, Ma L, et al. Indigenous bacteria from the gut microbiota regulate host serotonin biosynthesis. Cell. 2015;161(2):264-76. https://doi.org/10.1016/j.cell.2015.02.047.

25. Yarandi SS, Kulkarni S, Saha M, Sylvia KE, Sears CL, Pasricha PJ. Intestinal bacteria maintain adult enteric nervous system and nitrergic neurons via Toll-like receptor 2-induced neurogenesis in mice. Gastroenterology. 2020; 159:200-213.e208.

26. Yoon H, Schaubeck M, Lagkouvardos I, Blesl A, Heinzlmeir S, Hahne H, et al. Increased pancreatic protease activity in response to antibiotics impairs gut barrier and triggers colitis. Cell Mol Gastroenterol Hepatol. 2018;6:370-388.e373.

27. van Tilburg BE, Pettersen VK, Gutierrez MW, Laforest-Lapointe I, Jendzjowsky NG, Cavin J-B, et al. Intestinal fungi are causally implicated in microbiome assembly and immune development in mice. Nat Commun. 2020;11(1): 2577. https://doi.org/10.1038/s41467-020-16431-1.

28. Parker EA, Roy T, D'Adamo CR, Wieland LS. Probiotics and gastrointestinal conditions: an overview of evidence from the Cochrane Collaboration. Nutrition. 2018;45:125-134.e111. 
29. Ooijevaar RE, Terveer EM, Verspaget HW, Kuijper EJ, Keller JJ. Clinical application and potential of fecal microbiota transplantation. Annu Rev Med. 2019;70(1):335-51. https://doi.org/10.1146/annurev-med-111717-122 956.

30. Akira S, Takeda K. Toll-like receptor signalling. Nat Rev Immunol. 2004;4(7): 499-511. https://doi.org/10.1038/nri1391.

31. Burgueño JF, Abreu MT. Epithelial Toll-like receptors and their role in gut homeostasis and disease. Nat Rev Gastroenterol Hepatol. 2020;17(5):263-78. https://doi.org/10.1038/s41575-019-0261-4

32. Brun P, Giron MC, Qesari M, Porzionato A, Caputi V, Zoppellaro C, et al. Tolllike receptor 2 regulates intestinal inflammation by controlling integrity of the enteric nervous system. Gastroenterology. 2013;145(6):1323-33. https:// doi.org/10.1053/j.gastro.2013.08.047.

33. Barajon I, Serrao G, Arnaboldi F, Opizzi E, Ripamonti G, Balsari A, et al. Tolllike receptors 3, 4, and 7 are expressed in the enteric nervous system and dorsal root ganglia. J Histochem Cytochem. 2009;57(11):1013-23. https://doi. org/10.1369/jhc.2009.953539.

34. Soret R, Chevalier J, De Coppet P, Poupeau G, Derkinderen P, Segain JP, et al. Short-chain fatty acids regulate the enteric neurons and control gastrointestinal motility in rats. Gastroenterology. 2010;138(5):1772-82. https://doi.org/10.1053/j.gastro.2010.01.053.

35. Vincent AD, Wang XY, Parsons SP, Khan WI, Huizinga JD. Abnormal absorptive colonic motor activity in germ-free mice is rectified by butyrate, an effect possibly mediated by mucosal serotonin. Am J Physiol Gastrointest Liver Physiol. 2018;315(5):G896-g907. https://doi.org/10.1152/ajpgi.00237.201 7.

36. Reigstad CS, Salmonson CE, Rainey JF, Szurszewski JH, Linden DR, Sonnenburg $\mathrm{J}$, et al. Gut microbes promote colonic serotonin production through an effect of short-chain fatty acids on enterochromaffin cells. FASEB J. 2015;29(4):1395-403. https://doi.org/10.1096/fj.14-259598.

37. Esquerre N, Basso L, Defaye M, Vicentini FA, Cluny N, Bihan D, et al. Colitisinduced microbial perturbation promotes postinflammatory visceral hypersensitivity. Cell Mol Gastroenterol Hepatol. 2020;10(2):225-44. https:// doi.org/10.1016/j.jcmgh.2020.04.003.

38. Miller KA, Vicentini FA, Hirota SA, Sharkey KA, Wieser ME. Antibiotic treatment affects the expression levels of copper transporters and the isotopic composition of copper in the colon of mice. Proc Natl Acad Sci U S A. 2019;116(13):5955-60. https://doi.org/10.1073/pnas.1814047116.

39. Donaldson GP, Lee SM, Mazmanian SK. Gut biogeography of the bacterial microbiota. Nat Rev Microbiol. 2016;14(1):20-32. https://doi.org/10.1038/ nrmicro3552.

40. Mallon BS, Shick HE, Kidd GJ, Macklin WB. Proteolipid promoter activity distinguishes two populations of NG2-positive cells throughout neonatal cortical development. J Neurosci. 2002;22(3):876-85. https://doi.org/10.1523/ JNEUROSCI.22-03-00876.2002

41. Rao M, Nelms BD, Dong L, Salinas-Rios V, Rutlin M, Gershon MD, et al. Enteric glia express proteolipid protein 1 and are a transcriptionally unique population of glia in the mammalian nervous system. Glia. 2015;63(11): 2040-57. https://doi.org/10.1002/glia.22876.

42. Sharkey KA. Emerging roles for enteric glia in gastrointestinal disorders. J Clin Investig. 2015;125(3):918-25. https://doi.org/10.1172/JCl76303.

43. Kulkarni S, Micci MA, Leser J, Shin C, Tang SC, Fu YY, et al. Adult enteric nervous system in health is maintained by a dynamic balance between neuronal apoptosis and neurogenesis. Proc Natl Acad Sci U S A. 2017; 114(18):E3709-e3718. https://doi.org/10.1073/pnas.1619406114.

44. Belkind-Gerson J, Graham HK, Reynolds J, Hotta R, Nagy N, Cheng L, et al. Colitis promotes neuronal differentiation of Sox $2+$ and PLP1+ enteric cells. Sci Rep. 2017;7(1):2525. https://doi.org/10.1038/s41598-017-02890-y.

45. Belkind-Gerson J, Hotta R, Nagy N, Thomas AR, Graham H, Cheng L, et al. Colitis induces enteric neurogenesis through a 5-HT4-dependent mechanism. Inflamm Bowel Dis. 2015;21(4):870-8. https://doi.org/10.1097/ MIB.0000000000000326.

46. Heanue TA, Pachnis V. Prospective identification and isolation of enteric nervous system progenitors using Sox2. Stem Cells. 2011;29(1):128-40. https://doi.org/10.1002/stem.557.

47. Muller PA, Koscsó B, Rajani GM, Stevanovic K, Berres ML, Hashimoto D, et al. Crosstalk between muscularis macrophages and enteric neurons regulates gastrointestinal motility. Cell. 2014;158(2):300-13. https://doi.org/10.1016/j. cell.2014.04.050.

48. Smith PM, Howitt MR, Panikov N, Michaud M, Gallini CA, Bohlooly YM, et al. The microbial metabolites, short-chain fatty acids, regulate colonic Treg cell homeostasis. Science. 2013;341(6145):569-73. https://doi.org/10.1126/ science.1241165.

49. Husebye $E$, Hellström PM, Sundler F, Chen J, Midtvedt T. Influence of microbial species on small intestinal myoelectric activity and transit in germ-free rats. Am J Physiol Gastrointest Liver Physiol. 2001;280(3):G368-80. https://doi.org/10.1152/ajpgi.2001.280.3.G368.

50. Kashyap PC, Marcobal A, Ursell LK, Larauche M, Duboc H, Earle KA, et al. Complex interactions among diet, gastrointestinal transit, and gut microbiota in humanized mice. Gastroenterology. 2013;144(5):967-77. https://doi.org/10.1053/j.gastro.2013.01.047.

51. Lomasney KW, Houston A, Shanahan F, Dinan TG, Cryan JF, Hyland NP. Selective influence of host microbiota on CAMP-mediated ion transport in mouse colon. Neurogastroenterol Motil. 2014;26(6):887-90. https://doi.org/1 $0.1111 / \mathrm{nmo} .12328$.

52. Markle JG, Frank DN, Mortin-Toth S, Robertson CE, Feazel LM, RolleKampczyk U, et al. Sex differences in the gut microbiome drive hormonedependent regulation of autoimmunity. Science. 2013;339(6123):1084-8. https://doi.org/10.1126/science.1233521.

53. Delungahawatta T, Amin JY, Stanisz AM, Bienenstock J, Forsythe P, Kunze WA. Antibiotic driven changes in gut motility suggest direct modulation of enteric nervous system. Front Neurosci. 2017;11:588. https://doi.org/10.3389/ fnins.2017.00588.

54. Muller PA, Matheis F, Schneeberger M, Kerner Z, Jové V, Mucida D. Microbiota-modulated CART+ enteric neurons autonomously regulate blood glucose. Science. 2020;370(6514):314-21. https://doi.org/10.1126/ science.abd6176.

55. Suez J, Zmora N, Zilberman-Schapira G, Mor U, Dori-Bachash M, Bashiardes S, et al. Post-antibiotic gut mucosal microbiome reconstitution is impaired by probiotics and improved by autologous FMT. Cell. 2018;174:1406-1423.e1416.

56. Rakoff-Nahoum S, Paglino J, Eslami-Varzaneh F, Edberg S, Medzhitov R. Recognition of commensal microflora by toll-like receptors is required for intestinal homeostasis. Cell. 2004;118(2):229-41. https://doi.org/10.1016/j. cell.2004.07.002

57. Suzuki T, Yoshida S, Hara H. Physiological concentrations of short-chain fatty acids immediately suppress colonic epithelial permeability. Br J Nutr. 2008; 100(2):297-305. https://doi.org/10.1017/S0007114508888733.

58. Arpaia N, Campbell C, Fan X, Dikiy S, van der Veeken J, de Roos P, et al. Metabolites produced by commensal bacteria promote peripheral regulatory T-cell generation. Nature. 2013;504(7480):451-5. https://doi.org/1 0.1038/nature12726.

59. Neunlist M, Toumi F, Oreschkova T, Denis M, Leborgne J, Laboisse CL, et al. Human ENS regulates the intestinal epithelial barrier permeability and a tight junction-associated protein ZO-1 via VIPergic pathways. Am J Physiol Gastrointest Liver Physiol. 2003;285(5):G1028-36. https://doi.org/10.1152/a jpgi.00066.2003.

60. McVey Neufeld KA, Perez-Burgos A, Mao YK, Bienenstock J, Kunze WA. The gut microbiome restores intrinsic and extrinsic nerve function in germ-free mice accompanied by changes in calbindin. Neurogastroenterol Motil. 2015; 27(5):627-36. https://doi.org/10.1111/nmo.12534.

61. De Vadder F, Grasset E, Mannerås Holm L, Karsenty G, Macpherson AJ, Olofsson LE, et al. Gut microbiota regulates maturation of the adult enteric nervous system via enteric serotonin networks. Proc Natl Acad Sci U S A. 2018;115(25):6458-63. https://doi.org/10.1073/pnas.1720017115.

62. Laranjeira C, Sandgren K, Kessaris N, Richardson W, Potocnik A, Vanden Berghe $P$, et al. Glial cells in the mouse enteric nervous system can undergo neurogenesis in response to injury. J Clin Investig. 2011;121(9):3412-24. https://doi.org/10.1172/JCI58200.

63. Gonçalves JT, Schafer ST, Gage FH. Adult neurogenesis in the hippocampus: from stem cells to behavior. Cell. 2016;167(4):897-914. https://doi.org/10.101 6/j.cell.2016.10.021.

64. Joseph NM, He S, Quintana E, Kim Y-G, Núñez G, Morrison SJ. Enteric glia are multipotent in culture but primarily form glia in the adult rodent gut. J Clin Investig. 2011;121(9):3398-411. https://doi.org/10.1172/JCI58186.

65. Suárez-Rodríguez R, Belkind-Gerson J. Cultured nestin-positive cells from postnatal mouse small bowel differentiate ex vivo into neurons, glia, and smooth muscle. Stem Cells. 2004;22(7):1373-85. https://doi.org/10.1634/ stemcells.2003-0049.

66. Takaki M, Goto K, Kawahara I, Nabekura J. Activation of 5-HT4 receptors facilitates neurogenesis of injured enteric neurons at an anastomosis in the lower gut. J Smooth Muscle Res. 2015;51(0):82-94. https://doi.org/10.1540/ jsmr.51.82. 
67. Gershon MD. Behind an enteric neuron there may lie a glial cell. J Clin Invest. 2011;121(9):3386-9. https://doi.org/10.1172/JCl59573.

68. Belkind-Gerson J, Carreon-Rodriguez A, Benedict LA, Steiger C, Pieretti A, Nagy $\mathrm{N}$, et al. Nestin-expressing cells in the gut give rise to enteric neurons and glial cells. Neurogastroenterol Motil. 2013;25:61-69.e67.

69. Matheis F, Muller PA, Graves CL, Gabanyi I, Kerner ZJ, Costa-Borges D, et al. Adrenergic signaling in muscularis macrophages limits infection-induced neuronal loss. Cell. 2020;180:64-78.e16.

70. Obata Y, Pachnis $V$. The effect of microbiota and the immune system on the development and organization of the enteric nervous system. Gastroenterology. 2016;151(5):836-44. https://doi.org/10.1053/j.gastro.2016. 07.044.

71. Hyland NP, Cryan JF. Microbe-host interactions: influence of the gut microbiota on the enteric nervous system. Dev Biol. 2016;417(2):182-7. https://doi.org/10.1016/j.ydbio.2016.06.027.

72. Caputi V, Marsilio I, Cerantola S, Roozfarakh M, Lante I, Galuppini F, et al. Toll-like receptor 4 modulates small intestine neuromuscular function through nitrergic and purinergic pathways. Front Pharmacol. 2017;8:350. https://doi.org/10.3389/fphar.2017.00350.

73. Li ZX, Li QY, Qiao J, Lu CZ, Xiao BG. Granulocyte-colony stimulating factor is involved in low-dose LPS-induced neuroprotection. Neurosci Lett. 2009; 465(2):128-32. https://doi.org/10.1016/j.neulet.2009.08.069.

74. Schuster A, Klotz M, Schwab T, Di Liddo R, Bertalot T, Schrenk S, et al. Maintenance of the enteric stem cell niche by bacterial lipopolysaccharides? Evidence and perspectives. J Cell Mol Med. 2014;18(7):1429-43. https://doi. org/10.1111/jcmm.12292

75. Rolls A, Shechter R, London A, Ziv Y, Ronen A, Levy R, et al. Toll-like receptors modulate adult hippocampal neurogenesis. Nat Cell Biol. 2007; 9(9):1081-8. https://doi.org/10.1038/ncb1629.

76. Liu M-T, Kuan Y-H, Wang J, Hen R, Gershon MD. 5-HT4 receptor-mediated neuroprotection and neurogenesis in the enteric nervous system of adult mice. J Neurosci. 2009;29(31):9683-99. https://doi.org/10.1523/ JNEUROSCI.1145-09.2009.

77. Nøhr MK, Pedersen MH, Gille A, Egerod KL, Engelstoft MS, Husted AS, et al. GPR41/FFAR3 and GPR43/FFAR2 as cosensors for short-chain fatty acids in enteroendocrine cells vs FFAR3 in enteric neurons and FFAR2 in enteric leukocytes. Endocrinology. 2013;154(10):3552-64. https://doi.org/10.1210/ en.2013-1142.

78. Hayes CL, Dong J, Galipeau HJ, Jury J, McCarville J, Huang X, et al. Commensal microbiota induces colonic barrier structure and functions that contribute to homeostasis. Sci Rep. 2018;8(1):14184. https://doi.org/10.1038/ s41598-018-32366-6.

79. Guo S, Nighot M, Al-Sadi R, Alhmoud T, Nighot P, Ma TY. Lipopolysaccharide regulation of intestinal tight junction permeability is mediated by TLR4 signal transduction pathway activation of FAK and MyD88. J Immunol. 2015;195(10):4999-5010. https://doi.org/10.4049/ jimmunol.1402598.

80. Tulstrup MV-L, Christensen EG, Carvalho V, Linninge C, Ahrné S, Højberg O, et al. Antibiotic treatment affects intestinal permeability and gut microbial composition in Wistar rats dependent on antibiotic class. PloS One. 2015; 10(12):e0144854. https://doi.org/10.1371/journal.pone.0144854.

81. Reikvam DH, Erofeev A, Sandvik A, Grcic V, Jahnsen FL, Gaustad P, et al. Depletion of murine intestinal microbiota: effects on gut mucosa and epithelial gene expression. PLoS One. 2011;6(3):e17996. https://doi.org/10.13 71/journal.pone.0017996.

82. Erny D, Hrabě de Angelis AL, Jaitin D, Wieghofer P, Staszewski O, David E, et al. Host microbiota constantly control maturation and function of microglia in the CNS. Nat Neurosci. 2015;18(7):965-77. https://doi.org/10.103 8/nn.4030.

83. Colombo AV, Sadler RK, Llovera G, Singh V, Roth S, Heindl S, et al. Microbiotaderived short chain fatty acids modulate microglia and promote $A \beta$ plaque deposition. Elife. 2021;10:e59826. https://doi.org/10.7554/eLife.59826.

84. Barman M, Unold D, Shifley K, Amir E, Hung K, Bos N, et al. Enteric salmonellosis disrupts the microbial ecology of the murine gastrointestinal tract. Infect Immun. 2008;76(3):907-15. https://doi.org/10.1128/IAI.01432-07.

\section{Publisher's Note}

Springer Nature remains neutral with regard to jurisdictional claims in published maps and institutional affiliations. 